\title{
A molecular line study of the filamentary infrared dark cloud G304.74+01.32^
}

\begin{abstract}
O. Miettinen
Department of Physics, PO Box 64, 00014 University of Helsinki, Finland

e-mail: oskari .miettinen@helsinki.fi

Received 30 November 2011 / Accepted 14 February 2012

ABSTRACT

Context. Infrared dark clouds (IRDCs) are promising sites to study the earliest formation stages of stellar clusters and high-mass stars, and the physics of molecular-cloud formation and fragmentation.

Aims. We attempt to improve our understanding of the physical and chemical properties of the filamentary IRDC G304.74+01.32 (hereafter, G304.74). In particular, we investigate the kinematical and dynamical state of the cloud and clumps within it, and the amount of CO depletion.

Methods. All of the submillimetre peak positions in the cloud identified from our previous LABOCA 870- $\mu$ m map were observed in $\mathrm{C}^{17} \mathrm{O}(2-1)$ with APEX. These are the first line observations along the whole filament that have been made so far. Selected positions were also observed in the ${ }^{13} \mathrm{CO}(2-1), \mathrm{SiO}(5-4)$, and $\mathrm{CH}_{3} \mathrm{OH}\left(5_{k}-4_{k}\right)$ transitions at $\sim 1 \mathrm{~mm}$.

Results. The $\mathrm{C}^{17} \mathrm{O}$ lines were detected towards all target positions at similar radial velocities. $\mathrm{CO}$ does not appear to be significantly depleted in the clumps, the largest depletion factors being only about 2 . Two to three methanol $5_{k}-4_{k}$ lines near $\sim 241.8 \mathrm{GHz}$ were detected towards all selected positions, whereas $\mathrm{SiO}(5-4)$ was seen in only one of these positions, namely SMM 3 . In the band covering $\mathrm{SiO}(5-4)$, we also detected the $\mathrm{DCN}(3-2)$ line towards SMM 3. The ${ }^{13} \mathrm{CO}(2-1)$ lines display blue asymmetric profiles, which are indicative of large-scale infall motions. The clumps show transonic to supersonic non-thermal motions, and a virial-parameter analysis suggests that most of them are gravitationally bound. The external pressure may also play a non-negligible role in the dynamics. Our analysis suggests that the fragmentation of the filament into clumps is caused by a "sausage"-type instability, in agreement with results from other IRDCs.

Conclusions. The uniform $\mathrm{C}^{17} \mathrm{O}$ radial velocities along the $\mathrm{G} 304.74$ cloud shows that it is a coherent filamentary structure. Although the clumps appear to be gravitationally bound, the ambient turbulent ram pressure may be an important factor in the cloud dynamics. This is qualitatively consistent with our earlier suggestion that the filament was formed by converging supersonic turbulent flows. The poloidal magnetic field could resist the radial cloud collapse, which conforms to the low infall velocites that we derived. The cloud may be unable to form high-mass stars based on the mass-size threshold. The star-formation activity in the cloud, such as outflows, is likely responsible for the release of $\mathrm{CO}$ from the icy grain mantles back into the gas phase. Shocks related to outflows may also have injected $\mathrm{CH}_{3} \mathrm{OH}, \mathrm{SiO}$, and DCN into the gas-phase in SMM 3 .
\end{abstract}

Key words. ISM: abundances - ISM: molecules - ISM: clouds - stars: formation - ISM: individual objects: G304.74+01.32 radio lines: ISM

\section{Introduction}

Infrared dark clouds (IRDCs) are seen as dark absorption features against the Galactic background radiation, particularly at mid-infrared wavelengths (Pérault et al. 1996; Egan et al. 1998; Simon et al. 2006; Peretto \& Fuller 2009). Since their discovery in the mid to late nineties, and after the first detailed studies (Carey et al. 1998, 2000), IRDCs have been the subject of great research interest (e.g., Teyssier et al. 2002; Johnstone et al. 2003; Rathborne et al. 2005, 2006; Pillai et al. 2006; Beuther \& Sridharan 2007; Sakai et al. 2008; Ragan et al. 2009; Jiménez-Serra et al. 2010; Leurini et al. 2011; Kainulainen et al. $2011 \mathrm{~b}$, and many more). One reason for this widespread study of IRDCs is that they are found to be promising targets for the study of the initial conditions and early stages of high-mass

* This publication is based on data acquired with the Atacama Pathfinder EXperiment (APEX) under programmes 083.F-9302A and 087.F-9318A. APEX is a collaboration between the Max-PlanckInstitut für Radioastronomie, the European Southern Observatory, and the Onsala Space Observatory. star $\left(>8 M_{\odot}\right)$ and stellar cluster formation, both of which are quite poorly known compared to the formation of solar-type stars. Moreover, IRDCs are interesting objects for the studies of molecular-cloud formation and their fragmentation into clumps and cores (e.g., Miettinen \& Harju 2010, hereafter Paper I; Jackson et al. 2010; Wang et al. 2011). Infrared dark clouds are often found to be filamentary in shape, resembling the morphologies seen in numerical studies of cloud formation (e.g., Nakajima \& Hanawa 1996; Ballesteros-Paredes et al. 1999; Klessen \& Burkert 2000; Klessen 2001; Heitsch et al. 2008, 2011). On the other hand, theories and models of the instability and fragmentation of filamentary, or cylindrical, gas structures have been extensively studied in the past (e.g., Chandrasekhar \& Fermi 1953; Stodólkiewicz 1963; Ostriker 1964; Bastien 1983; Inutsuka \& Miyama 1992; Curry 2000). The filamentary IRDCs are wellsuited to testing these theories/models, such as their predictions about the preferred fragmentation length-scales.

The target cloud of the present study is the IRDC G304.74+01.32 (hereafter, G304.74). We recently mapped this IRDC in the $870-\mu \mathrm{m}$ dust continuum emission 


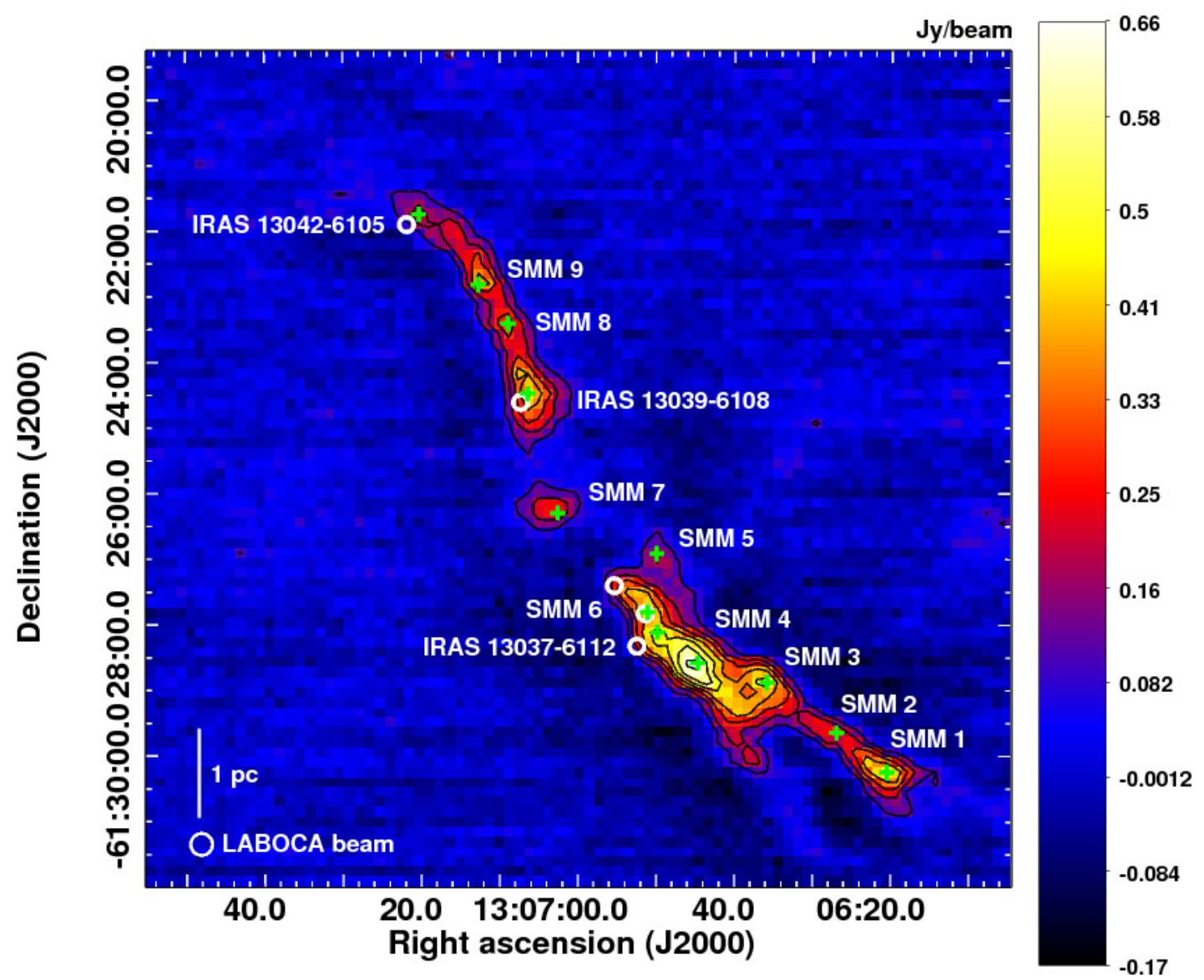

Fig. 1. LABOCA 870- $\mu \mathrm{m}$ image of the IRDC G304.74. The image is shown with linear scaling, and the colour bar indicates the flux density in units of Jy beam ${ }^{-1}$. The rms level is $0.03 \mathrm{Jy} \mathrm{beam}^{-1}(1 \sigma)$. The first contour and the separation between contours is $3 \sigma$. The green plus signs show the submm peak positions, which were used as the line-observation target positions in the present study. The white circles indicate the nominal catalogue positions of the MSX 8- $\mu \mathrm{m}$ sources. The 1-pc scale bar and the effective LABOCA beam HPBW $\left(\sim 20^{\prime \prime}\right)$ are shown in the bottom left (modified from Paper I).

with the LABOCA bolometer on APEX (Paper I). The cloud was found to have a integral-shaped/“hub-filament"-kind of structure (Myers 2009), and it was resolved into twelve clumps with the two-dimensional clumpfind algorithm (Williams et al. 1994). Four of the clumps were found to be associated with MSX $8-\mu \mathrm{m}$ point-like sources, indicating embedded star formation. Moreover, three of these MSX-bright clumps are associated with IRAS point sources (the IRAS sources 13037-6112, 13039-6108, and 13042-6105; hereafter, IRAS 13037 etc.). Eight clumps in G304.74 were found to be dark in the MSX 8- $\mu \mathrm{m}$ image. The clump masses were estimated to be in the range $\sim 40-220 M_{\odot}$ (assuming a dust temperature of 15 or $22 \mathrm{~K}$ ), within the effective radii of $\sim 0.3-0.5 \mathrm{pc}$. Unfortunately, G304.74 is not covered by the Spitzer infrared maps, which "only" cover the Galactic latitudes $|b| \leq 1^{\circ}$. Our 870- $\mu$ m LABOCA map of G304.74 is shown in Fig. 1 (modified from Fig. 1 of Paper I). We also refer to Fig. 2 of Paper I for the MSX $8-\mu \mathrm{m}$ image of the region overlaid with LABOCA contours.

To our knowledge, besides the IRDC identification of Simon et al. (2006) and our Paper I, the only studies of G304.74, or a clump within it, are those of Fontani et al. (2005) and Beltrán et al. (2006). The latter authors performed SIMBA 1.2mm dust continuum mapping of the cloud, and identified eight clumps, including the MSX-bright sources (see our Paper I for more details). Fontani et al. (2005) carried out pointed CS and $\mathrm{C}^{17} \mathrm{O}$ observations towards one source in $\mathrm{G} 304.74$, namely IRAS 13039. By employing the Galactic rotation curve of Brand \& Blitz (1993) and the radial velocity $-26.2 \mathrm{~km} \mathrm{~s}^{-1}$ derived from CS observations, Fontani et al. (2005) determined the source distance of $2.44 \mathrm{kpc}$. As discussed by Beuther et al. (2011), G304.74 constitutes a small part of the kpc-distance cloud complexes, which form a portion of the Coalsack dark cloud in the plane of the sky.

In the present paper, we present the first molecular-line observations along the whole filamentary structure of G304.74. These data are used to examine the basic physical properties of the cloud, such as the gas kinematics and dynamical state of the clumps. The cloud's kinematic distance, and the distancedependent parameters presented in Paper I, will also be revised. This study will also address some chemical properties of the clumps. For example, we investigate the amount of CO depletion, which has only recently been studied in IRDCs (Zhang et al. 2009; Hernandez et al. 2011; Miettinen et al. 2011; Chen et al. 2011).

The rest of this paper is structured as follows. The observations and data-reduction procedures are described in Sect. 2. The direct observational results are presented in Sect. 3. In Sect. 4/Appendix A, we describe the analysis and present the results of the physical and chemical properties of the cloud/clumps. Discussion of our results is presented in Sect. 5, and in Sect. 6 we summarise the main conclusions of this study. 
Table 1. Source list.

\begin{tabular}{|c|c|c|c|c|c|c|c|}
\hline Source & $\begin{array}{c}\alpha_{2000.0} \\
{[\mathrm{~h}: \mathrm{m}: \mathrm{s}]}\end{array}$ & $\begin{array}{l}\delta_{2000.0} \\
{\left[:^{\circ}::^{\prime \prime}\right]}\end{array}$ & $\begin{array}{c}R \\
{[\mathrm{pc}]}\end{array}$ & $\begin{array}{c}M \\
{\left[M_{\odot}\right]}\end{array}$ & $\begin{array}{c}N\left(\mathrm{H}_{2}\right) \\
{\left[10^{22} \mathrm{~cm}^{-2}\right]}\end{array}$ & $\begin{array}{c}\left\langle n\left(\mathrm{H}_{2}\right)\right\rangle \\
{\left[10^{4} \mathrm{~cm}^{-3}\right]}\end{array}$ & MSX $8 \mu \mathrm{m}$ \\
\hline SMM 1 & 130620.5 & -613015 & $0.44 \pm 0.12$ & $121 \pm 92$ & $2.1 \pm 1.2$ & $0.7 \pm 0.5$ & dark \\
\hline SMM 2 & 130626.9 & -612939 & $0.34 \pm 0.09$ & $61 \pm 46$ & $1.4 \pm 0.8$ & $0.7 \pm 0.5$ & dark \\
\hline SMM 3 & 130635.8 & -612853 & $0.52 \pm 0.13$ & $206 \pm 156$ & $2.3 \pm 1.3$ & $0.7 \pm 0.5$ & dark \\
\hline SMM 4 & 130644.7 & -612835 & $0.47 \pm 0.12$ & $248 \pm 188$ & $3.0 \pm 1.7$ & $1.1 \pm 0.8$ & dark \\
\hline IRAS 13037-6112 & 130649.8 & -612807 & $0.32 \pm 0.08$ & $54 \pm 28$ & $1.2 \pm 0.1$ & $0.8 \pm 0.4$ & point \\
\hline SMM 5 & 130649.9 & -612655 & $0.32 \pm 0.08$ & $42 \pm 32$ & $0.9 \pm 0.5$ & $0.6 \pm 0.4$ & dark \\
\hline SMM 6 & 130651.1 & -612749 & $0.37 \pm 0.10$ & $109 \pm 83$ & $2.1 \pm 1.2$ & $1.0 \pm 0.8$ & point \\
\hline SMM 7 & 130702.6 & -612618 & $0.37 \pm 0.10$ & $55 \pm 41$ & $1.2 \pm 0.7$ & $0.5 \pm 0.4$ & dark \\
\hline IRAS 13039-6108 & 130706.4 & -612429 & $0.49 \pm 0.13$ & $88 \pm 46$ & $1.2 \pm 0.1$ & $0.3 \pm 0.2$ & point \\
\hline SMM 8 & 130708.9 & -612325 & $0.36 \pm 0.09$ & $67 \pm 50$ & $1.4 \pm 0.8$ & $0.7 \pm 0.5$ & dark \\
\hline SMM 9 & 130712.7 & -612249 & $0.39 \pm 0.10$ & $97 \pm 73$ & $1.7 \pm 1.0$ & $0.8 \pm 0.6$ & dark \\
\hline IRAS 13042-6105 & 130720.3 & -612145 & $0.33 \pm 0.09$ & $48 \pm 37$ & $1.0 \pm 0.5$ & $0.6 \pm 0.5$ & point \\
\hline
\end{tabular}

Notes. The clump physical properties are revised from those presented in Paper I (see Sect. 4). Columns (2) and (3) give the equatorial coordinates $\left[(\alpha, \delta)_{2000.0}\right]$ of the LABOCA peak position. Columns (4)-(7) list, respectively, the clump effective radius, mass, beam-averaged peak $\mathrm{H}_{2}$ column density, and the volume-averaged $\mathrm{H}_{2}$ number density. In the last column, we indicate whether the clump appears dark or bright in the MSX 8- $\mu \mathrm{m}$ image.

Table 2. Observed spectral-line transitions and observational parameters.

\begin{tabular}{|c|c|c|c|c|c|c|c|c|c|}
\hline Transition & $\begin{array}{c}v^{a} \\
{[\mathrm{MHz}]}\end{array}$ & $\begin{array}{c}\text { HPBW } \\
{\left[{ }^{\prime \prime}\right]}\end{array}$ & $\eta_{\mathrm{MB}}$ & $\begin{array}{l}T_{\text {sys }} \\
{[\mathrm{K}]}\end{array}$ & $\begin{array}{l}\text { PWV } \\
{[\mathrm{mm}]}\end{array}$ & $\begin{array}{l}\text { Channe } \\
{[\mathrm{kHz}]}\end{array}$ & $\begin{array}{l}\text { spacing }^{b} \\
{\left[\mathrm{~km} \mathrm{~s}^{-1}\right]}\end{array}$ & $\begin{array}{c}t_{\mathrm{int}} \\
{[\mathrm{min}]}\end{array}$ & $\begin{array}{c}\mathrm{rms} \\
{[\mathrm{mK}]}\end{array}$ \\
\hline $\mathrm{SiO}(5-4)$ & 217104.980 & 28.7 & 0.75 & $324-360$ & $0.7-1.2$ & 122.07 & 0.17 & 9.5 & $38-43$ \\
\hline $\operatorname{DCN}(3-2)^{c}$ & $217238.61^{c}$ & 28.7 & 0.75 & 337 & $0.8-0.9$ & 122.07 & 0.17 & 9.5 & 39 \\
\hline${ }^{13} \mathrm{CO}(2-1)$ & $220398.70056^{d}$ & 28.3 & 0.75 & $282-371$ & $0.8-1.0$ & 122.07 & 0.17 & 9.5 & $43-46$ \\
\hline $\mathrm{C}^{17} \mathrm{O}(2-1)$ & $224714.199^{e}$ & 27.8 & 0.75 & $392-418$ & $0.7-1.7$ & 122.07 & 0.16 & 2.6 & $81-102$ \\
\hline $\mathrm{CH}_{3} \mathrm{OH}\left(5_{-1,5}-4_{-1,4}\right)-\mathrm{E}$ & 241767.224 & 25.8 & 0.75 & $430-445$ & $0.4-0.8$ & 122.07 & 0.15 & $10.5-15.5$ & $39-49$ \\
\hline $\mathrm{CH}_{3} \mathrm{OH}\left(5_{0,5}-4_{0,4}\right)-\mathrm{A}^{+}$ & 241791.431 & $\cdots$ & $\ldots$ & $\ldots$ & $\ldots$ & $\ldots$ & $\ldots$ & $\ldots$ & $\ldots$ \\
\hline $\mathrm{CH}_{3} \mathrm{OH}\left(5_{4, *}-4_{4, *}\right)-\mathrm{A}^{+/-f}$ & 241806.508 & $\ldots$ & $\ldots$ & $\ldots$ & $\ldots$ & $\ldots$ & $\ldots$ & $\ldots$ & $\ldots$ \\
\hline
\end{tabular}

Notes. Columns (2)-(10) give the rest frequencies of the observed transitions ( $v$ ), the APEX beamsize (HPBW) and the main beam efficiency $\left(\eta_{\mathrm{MB}}\right)$ at the observed frequencies, the single-sideband system temperatures during the observations ( $T_{\mathrm{sys}}$ in $T_{\mathrm{MB}}$ scale, see text), the amount of precipitable water vapour (PWV), channel widths (both in $\mathrm{kHz}$ and $\mathrm{km} \mathrm{s}^{-1}$ ) of the original data, the on-source integration times per position $\left(t_{\text {int }}\right)$, and the $1 \sigma$ rms noise at the smoothed resolution. ${ }^{(a)}$ The rest frequencies were taken from the Cologne Database for Molecular Spectroscopy (CDMS; http://www .astro.uni-koeln.de/cdms/catalog) (Müller et al. 2005) unless otherwise stated. ${ }^{(b)}$ The original channel spacings. The final spectra were Hanning-smoothed by dividing the number of channels by two. ${ }^{(c)}$ The DCN(3-2) transition was additionally detected in the band covering the $\mathrm{SiO}(5-4)$ line. The quoted frequency refers to the strongest hyperfine component $F=4-3$. ${ }^{(d)}$ The frequency was taken from Cazzoli et al. (2004) and refers to the strongest hyperfine component $F=5 / 2-3 / 2$. $^{(e)}$ The frequency was taken from Ladd et al. (1998) and refers to the strongest hyperfine component $F=9 / 2-7 / 2$. ${ }^{(f)}$ A blend of $\mathrm{CH}_{3} \mathrm{OH}\left(5_{4,1}-4_{4,0}\right)-\mathrm{A}^{+}$and $\mathrm{CH}_{3} \mathrm{OH}\left(5_{4,2}-4_{4,1}\right)-\mathrm{A}^{-}$, which have the same frequency.

\section{Observations and data reduction}

The observations presented in this paper were made using the APEX 12-m telescope at Llano de Chajnantor (Chilean Andes). The telescope and its performance are described in the paper by Güsten et al. (2006). All the submm peak positions in G304.74 identified from our previous LABOCA $870-\mu \mathrm{m}$ map were observed in $\mathrm{C}^{17} \mathrm{O}(2-1)$, and selected positions in the southern part of the filament were observed in ${ }^{13} \mathrm{CO}(2-1)$, $\mathrm{SiO}(5-4)$, and $\mathrm{CH}_{3} \mathrm{OH}\left(5_{k}-4_{k}\right)$ with the Swedish Heterodyne Facility Instrument (SHeFI; Belitsky et al. 2007; Vassilev et al. 2008a). The target positions with their physical properties are listed in Table 1. The observed transitions and observational parameters are listed in Table 2. The observations took place on 18-19 and 26-28 May 2011. As a frontend to the observations, we used the APEX-1 receiver of the SHeFI (Vassilev et al. 2008b). The backend to all observations was the Fast Fourier Transfrom Spectrometer (FFTS; Klein et al. 2006) with a $1 \mathrm{GHz}$ bandwidth divided into 8192 channels.

The observations were performed in the wobbler-switching mode with a $150^{\prime \prime}$ azimuthal throw (symmetric offsets) and a chopping rate of $0.5 \mathrm{~Hz}$. The telescope pointing and focus corrections were checked by continuum scans of the planet Saturn, and the pointing was found to be better than $\sim 5^{\prime \prime}$. Calibration was done by means of the chopper-wheel technique, and the output intensity scale given by the system is $T_{\mathrm{A}}^{*}$, which represents the antenna temperature corrected for atmospheric attenuation. The observed intensities were converted to the main-beam brightness temperature scale by $T_{\mathrm{MB}}=T_{\mathrm{A}}^{*} / \eta_{\mathrm{MB}}$, where $\eta_{\mathrm{MB}}$ is the main beam efficiency. The absolute calibration uncertainty is estimated to be around $10 \%$.

The spectra were reduced using the CLASS90 programme of the IRAM's GILDAS software package ${ }^{1}$. The individual spectra were averaged and the resulting spectra were Hanning-smoothed in order to improve the signal-to-noise ratio of the data. A first- or third-order polynomial was applied to correct the baseline in the final spectra. The resulting $1 \sigma$ rms noise levels are $\sim 40-100 \mathrm{mK}$ at the smoothed resolutions.

The ${ }^{13} \mathrm{CO}(2-1)$ and $\mathrm{C}^{17} \mathrm{O}(2-1)$ rotational lines contain three and nine hyperfine (hf) components, respectively. We fitted these

${ }^{1}$ http://wWw.iram.fr/IRAMFR/GILDAS 

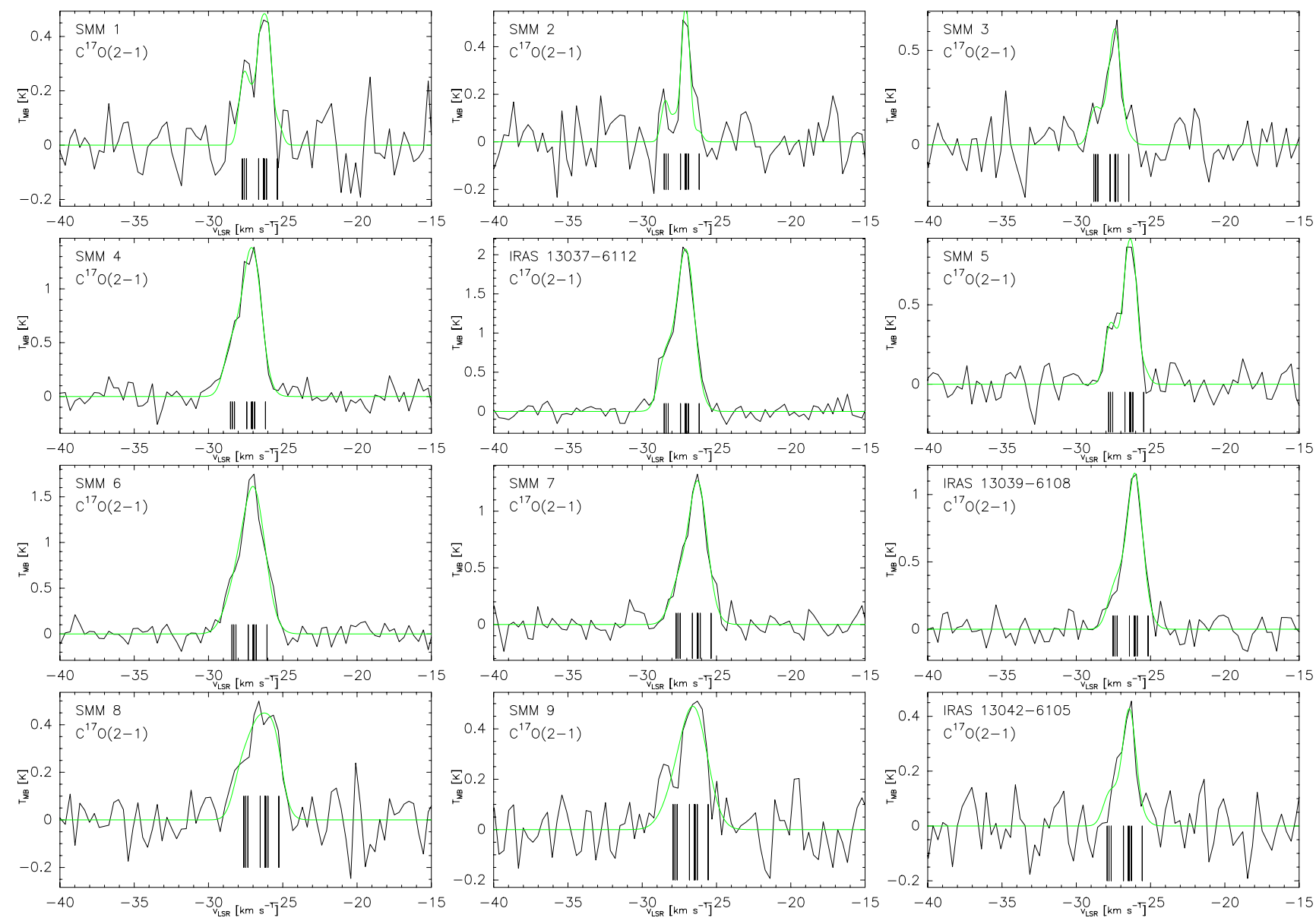

Fig. 2. Smoothed $\mathrm{C}^{17} \mathrm{O}(2-1)$ spectra. Overlaid on the spectra are the hf-structure fits. The relative velocities of individual hf components are indicated by vertical lines under the spectra.

hf structures using "method hfs" of CLASS90 to derive the LSR velocity $\left(v_{\text {LSR }}\right)$ of the emission, and full width at half maximum (FWHM) linewidth $(\Delta v)$. The hf-line fitting can also be used to derive the line optical thickness, $\tau$. However, in all spectra the hf components are blended together, thus the optical thickness could not be reliably determined. For the rest frequencies of the ${ }^{13} \mathrm{CO}(2-1)$ hf components, we used the values from Cazzoli et al. (2004; Table 2 therein), whereas those for $\mathrm{C}^{17} \mathrm{O}(2-1)$ were taken from Ladd et al. (1998; Table 6 therein).

\section{Observational results}

The smoothed $\mathrm{C}^{17} \mathrm{O}(2-1)$ spectra towards all target positions are shown in Fig. 2. The line was clearly detected towards all sources, but its hf components are mostly blended in all cases. Towards a few sources, such as SMM 2, the hf structure is only partially resolved, but not sufficiently well to derive the line optical thickness. Figures $3-5$ show the ${ }^{13} \mathrm{CO}(2-1)$, $\mathrm{SiO}(5-4)$, and $\mathrm{CH}_{3} \mathrm{OH}\left(5_{k}-4 k\right)$ spectra towards the observed five clumps in the southern part of the filament. All the ${ }^{13} \mathrm{CO}(2-1)$ lines show a blue asymmetric profile, i.e., the blueshifted peak is stronger than the redshifted peak, and there is a selfabsorption dip near the systemic velocity. The self-absorbed profiles of the ${ }^{13} \mathrm{CO}$ lines indicate that the lines are optically thick. Moreover, the lines show non-Gaussian line-wing emission. In particular, SMM 2 shows high-velocity blueshifted wing emission up to $-35 \mathrm{~km} \mathrm{~s}^{-1}$. The small "absorption"-like features seen in the ${ }^{13} \mathrm{CO}$ spectra of SMM 4 and IRAS 13037 are caused by weak emission in the off-source reference position (off-beam). The line $\mathrm{SiO}(5-4)$ was detected only towards SMM 3, though the line is also very weak towards this source. In addition, we detected the DCN(3-2) line towards SMM 3 in the frequency band covering $\mathrm{SiO}(5-4)$ (see Fig. 6). The detected $J=3-2$ rotational line of DCN is split into six hf components. In fitting this hf structure, we used the rest frequencies from the CDMS catalogue. The E-type methanol transition $\mathrm{CH}_{3} \mathrm{OH}\left(5_{-1,5}-4_{-1,4}\right)$-E and the A-type transition $\mathrm{CH}_{3} \mathrm{OH}\left(5_{0,5}-4_{0,4}\right)-\mathrm{A}^{+}$at $\sim 241.8 \mathrm{GHz}$ were detected towards all the observed clumps. Moreover, towards SMM 2, a blend of $\mathrm{CH}_{3} \mathrm{OH}\left(5_{4,1}-4_{4,0}\right)-\mathrm{A}^{+}$and $\mathrm{CH}_{3} \mathrm{OH}\left(5_{4,2}-4_{4,1}\right)-\mathrm{A}^{-}$was detected.

The spectral-line parameters derived from hf- and single Gaussian fits are given in Cols. (3)-(5) of Table 3. For the $\mathrm{SiO}(5-4)$ non-detections, we provide the $3 \sigma$ upper limit to the line intensity. The integrated line intensities listed in Col. (6) of Table 3 were computed over the velocity range given in square brackets in the corresponding column. The quoted uncertainties in $v_{\mathrm{LSR}}$ and $\Delta v$ are formal $1 \sigma$ fitting errors, whereas those in $T_{\mathrm{MB}}$ and $\int T_{\mathrm{MB}} \mathrm{d} v$ also include the $10 \%$ calibration uncertainty. In the last column, we give the estimated peak line optical-thicknesses, which are derived in Sect. 4.3/Appendix A.3.

\section{Analysis and results}

\subsection{Kinematic distance of G304.74+01.32}

The LSR velocities derived from $\mathrm{C}^{17} \mathrm{O}(2-1)$ lie in the range $v_{\text {LSR }} \in[-27.4,-26.1] \mathrm{km} \mathrm{s}^{-1}$, i.e., within $1.3 \mathrm{~km} \mathrm{~s}^{-1}$. This velocity coherence shows that the filamentary IRDC G304.74 is a 

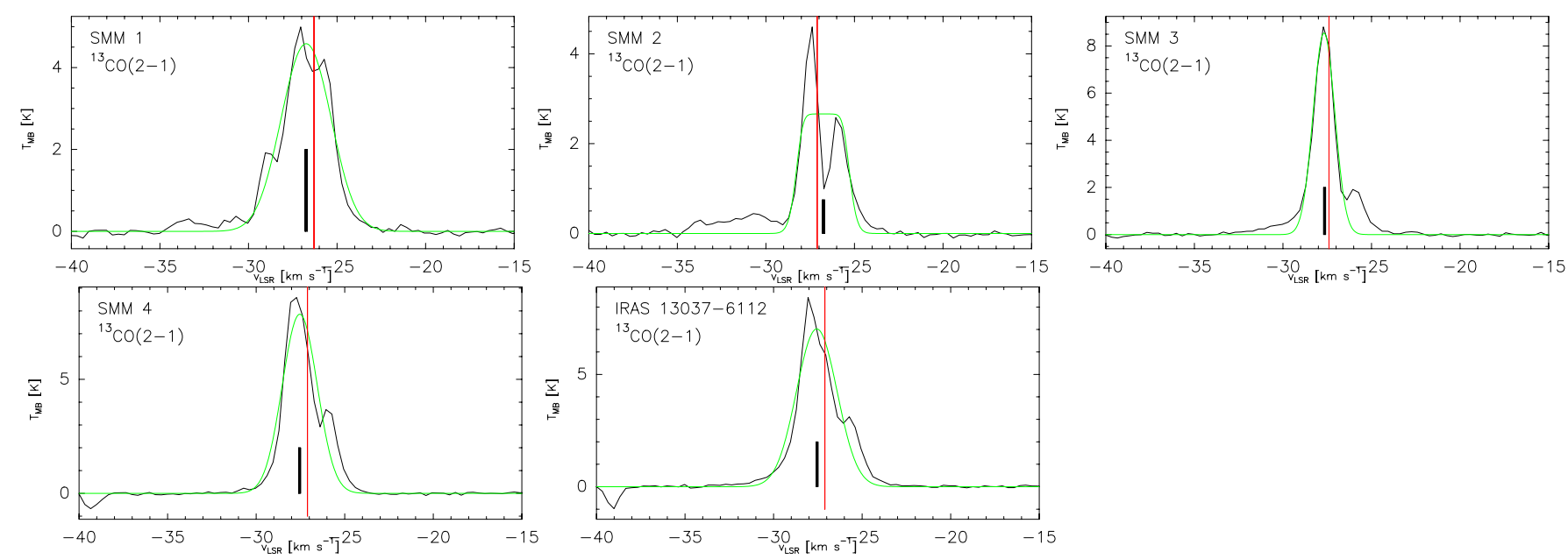

Fig. 3. Smoothed ${ }^{13} \mathrm{CO}(2-1)$ spectra overlaid with hf-structure fits. The relative velocities of individual hf components are indicated by vertical lines under the spectra. The red vertical line shows the systemic velocity derived from $\mathrm{C}^{17} \mathrm{O}(2-1)$.
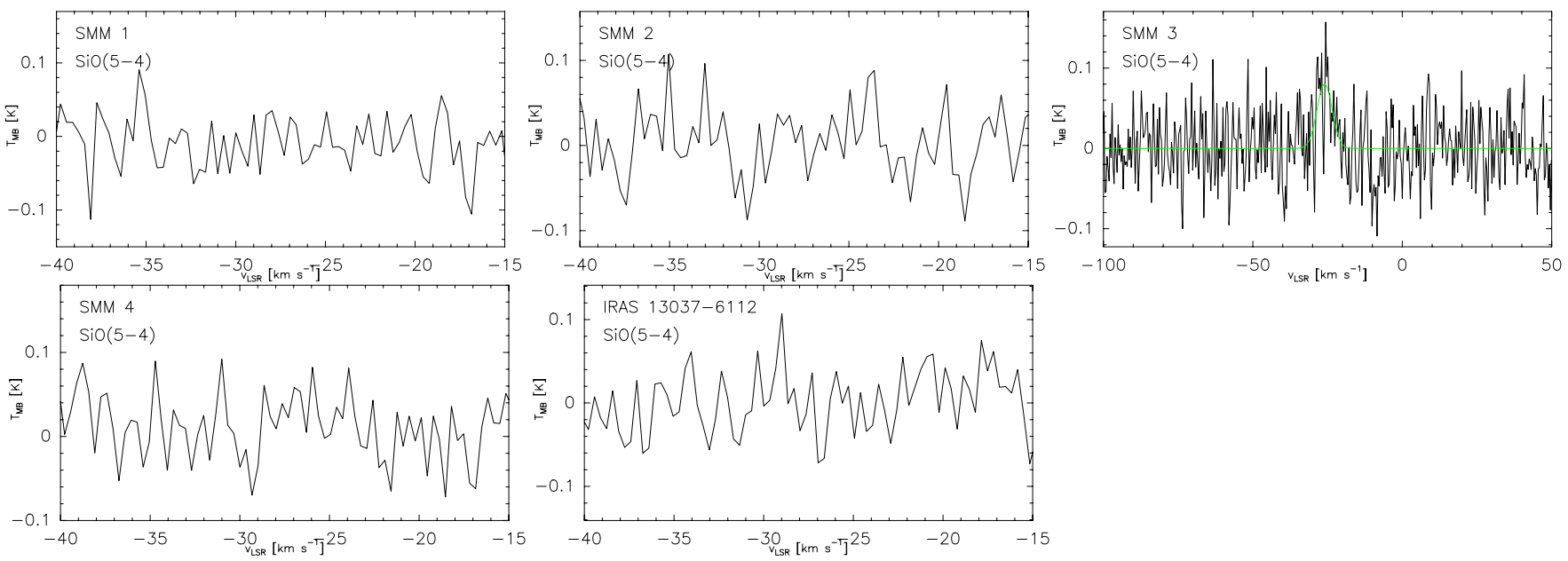

Fig. 4. Smoothed $\mathrm{SiO}(5-4)$ spectra. The line was detected only towards SMM 3 (overlaid with a Gaussian fit). Note that the spectrum towards SMM 3 is shown with a wider velocity range for reasons of clarity.
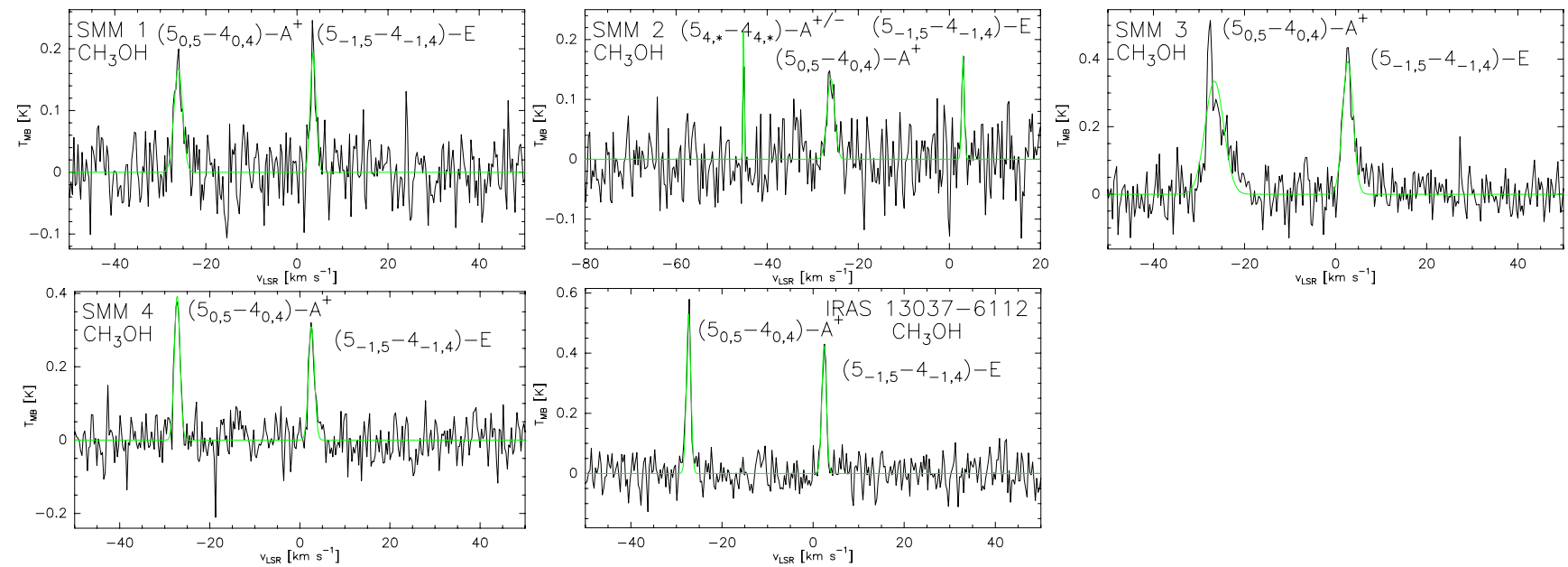

Fig. 5. Smoothed $\mathrm{CH}_{3} \mathrm{OH}\left(5_{k}-4_{k}\right)$ spectra with Gaussian fits overlaid. In the spectrum towards $\mathrm{SMM} 2$, the $\mathrm{CH}_{3} \mathrm{OH}\left(5_{4,1}-4_{4,0}\right)-\mathrm{A}^{+}$and $\mathrm{CH}_{3} \mathrm{OH}\left(5_{4,2}-\right.$ $\left.4_{4,1}\right)-\mathrm{A}^{-}$transitions are blended. Note that the velocity range shown is different for SMM 2 for reasons of clarity.

coherent structure, and not just a group of physically independent objects seen in projection on the plane of the sky.

The mean and median radial velocities derived from $\mathrm{C}^{17} \mathrm{O}(2-1)$ are -26.7 and $-26.5 \mathrm{~km} \mathrm{~s}^{-1}$, respectively. To revise the cloud kinematic distance, we adopt the mean velocity of $-26.7 \mathrm{~km} \mathrm{~s}^{-1}$, and employ the rotation curve of Reid et al. (2009). The resulting near kinematic distance, as expected to be appropriate for an IRDC seen in absorption, is 
Table 3. Spectral-line parameters.

\begin{tabular}{|c|c|c|c|c|c|c|}
\hline Source & Transition & $\begin{array}{c}v_{\mathrm{LSR}} \\
{\left[\mathrm{km} \mathrm{s}^{-1}\right]}\end{array}$ & $\begin{array}{c}\Delta v \\
{\left[\mathrm{~km} \mathrm{~s}^{-1}\right]}\end{array}$ & $\begin{array}{c}T_{\mathrm{MB}}{ }^{a} \\
{[\mathrm{~K}]}\end{array}$ & $\begin{array}{c}\int_{\left[\mathrm{K} \mathrm{km} \mathrm{s}^{-1}\right]} T_{\mathrm{MB}} \mathrm{d} v^{b} \\
\end{array}$ & $\tau_{0}$ \\
\hline \multirow{5}{*}{ SMM 1} & $\mathrm{SiO}(5-4)$ & & & $<0.13$ & & \\
\hline & ${ }^{13} \mathrm{CO}(2-1)$ & $-26.8 \pm 0.01$ & $3.18 \pm 0.01$ & $4.92 \pm 0.63(\mathrm{~B}) / 4.16 \pm 0.57(\mathrm{R})$ & $15.78 \pm 1.59[-30.13,-22.65]$ & $\cdots$ \\
\hline & $\mathrm{C}^{17} \mathrm{O}(2-1)$ & $-26.3 \pm 0.1$ & $0.68 \pm 0.13$ & $0.40 \pm 0.08$ & $0.87 \pm 0.14[-28.88,-25.37]$ & $0.04 \pm 0.02$ \\
\hline & $\mathrm{CH}_{3} \mathrm{OH}\left(5_{-1,5}-4_{-1,4}\right)-\mathrm{E}$ & $-26.5 \pm 0.1$ & $1.47 \pm 0.27$ & $0.19 \pm 0.04$ & $0.35 \pm 0.05[-28.24,-24.57]$ & $0.01 \pm 0.001-0.04 \pm 0.01$ \\
\hline & $\mathrm{CH}_{3} \mathrm{OH}\left(5_{0,5}-4_{0,4}\right)-\mathrm{A}^{+}$ & $-26.1 \pm 0.1$ & $2.22 \pm 0.37$ & $0.17 \pm 0.03$ & $0.39 \pm 0.06[-27.57,-23.23]$ & $0.01 \pm 0.001-0.03 \pm 0.01$ \\
\hline \multirow[t]{6}{*}{ SMM 2} & $\mathrm{SiO}(5-4)$ & $\ldots$ & $\ldots$ & $<0.12$ & & $\ldots$ \\
\hline & ${ }^{13} \mathrm{CO}(2-1)$ & $-26.8 \pm 0.03$ & $1.53 \pm 0.08$ & $4.57 \pm 0.51(\mathrm{~B}) / 2.51 \pm 0.4(\mathrm{R})$ & $8.47 \pm 0.87[-28.96,-23.11]$ & \\
\hline & $\mathrm{C}^{17} \mathrm{O}(2-1)$ & $-27.1 \pm 0.1$ & $0.59 \pm 0.19$ & $0.53 \pm 0.10$ & $0.63 \pm 0.10[-29.05,-25.66]$ & $0.05 \pm 0.03$ \\
\hline & $\mathrm{CH}_{3} \mathrm{OH}\left(5_{-1,5}-4_{-1,4}\right)-\mathrm{E}$ & $-27.0 \pm 0.1$ & $0.67 \pm 0.23$ & $0.17 \pm 0.03$ & $0.14 \pm 0.03[-28.31,-26.10]$ & $0.01 \pm 0.001-0.03 \pm 0.01$ \\
\hline & $\mathrm{CH}_{3} \mathrm{OH}\left(5_{0,5}-4_{0,4}\right)-\mathrm{A}^{+}$ & $-26.0 \pm 0.2$ & $1.98 \pm 0.46$ & $0.14 \pm 0.02$ & $0.30 \pm 0.06[-28.13,-23.53]$ & $0.01 \pm 0.01-0.03 \pm 0.01$ \\
\hline & $\mathrm{CH}_{3} \mathrm{OH}\left(5_{4, *}-4_{4, *}\right)-\mathrm{A}^{+/-c}$ & $-26.5 \pm 0.04$ & $0.30 \pm 0.80$ & $0.13 \pm 0.02$ & $0.17 \pm 0.03[-27.02,-25.55]$ & \\
\hline \multirow[t]{6}{*}{ SMM 3} & $\mathrm{SiO}(5-4)$ & $-26.0 \pm 0.5$ & $5.99 \pm 1.06$ & $0.08 \pm 0.05$ & $0.49 \pm 0.09[-32.02,-19.99]$ & $0.003 \pm 0.001-0.07 \pm 0.01$ \\
\hline & ${ }^{13} \mathrm{CO}(2-1)$ & $-27.7 \pm 0.01$ & $1.41 \pm 0.03$ & $8.81 \pm 0.93(\mathrm{~B}) / 1.91 \pm 0.35(\mathrm{R})$ & $14.69 \pm 1.48[-29.26,-24.91]$ & \\
\hline & $\mathrm{C}^{17} \mathrm{O}(2-1)$ & $-27.4 \pm 0.1$ & $0.84 \pm 0.35$ & $0.52 \pm 0.11$ & $0.91 \pm 0.15[-29.55,-25.75]$ & $0.05 \pm 0.03$ \\
\hline & $\mathrm{CH}_{3} \mathrm{OH}\left(5_{-1,5}-4_{-1,4}\right)-\mathrm{E}$ & $-27.3 \pm 0.1$ & $2.70 \pm 0.21$ & $0.39 \pm 0.09$ & $1.66 \pm 0.18[-29.75,-21.73]$ & $0.02 \pm 0.01-0.08 \pm 0.01$ \\
\hline & $\mathrm{CH}_{3} \mathrm{OH}\left(5_{0,5}-4_{0,4}\right)-\mathrm{A}^{+}$ & $-26.6 \pm 0.2$ & $4.82 \pm 0.60$ & $0.51 \pm 0.09$ & $1.85 \pm 0.24[-30.75,-18.38]$ & $0.03 \pm 0.01-0.10 \pm 0.01$ \\
\hline & $\mathrm{DCN}(3-2)$ & $-27.4 \pm 0.1$ & $1.09 \pm 0.27$ & $0.13 \pm 0.04$ & $0.19 \pm 0.05[-28.76,-26.00 ; 92.6 \%]$ & $0.01 \pm 0.001-0.11 \pm 0.01$ \\
\hline \multirow[t]{5}{*}{ SMM 4} & $\mathrm{SiO}(5-4)$ & $\ldots$ & $\ldots$ & $<0.11$ & & $\ldots$ \\
\hline & ${ }^{13} \mathrm{CO}(2-1)$ & $-27.6 \pm 0.01$ & $2.27 \pm 0.03$ & $8.94 \pm 0.93(\mathrm{~B}) / 3.32 \pm 0.42(\mathrm{R})$ & $20.04 \pm 2.01[-30.13,-23.91]$ & \\
\hline & $\mathrm{C}^{17} \mathrm{O}(2-1)$ & $-27.1 \pm 0.03$ & $1.20 \pm 0.15$ & $1.33 \pm 0.18$ & $2.80 \pm 0.30[-30.55,-25.54]$ & $0.14 \pm 0.07$ \\
\hline & $\mathrm{CH}_{3} \mathrm{OH}\left(5_{-1,5}-4_{-1,4}\right)-\mathrm{E}$ & $-27.4 \pm 0.1$ & $1.53 \pm 0.18$ & $0.31 \pm 0.04$ & $0.61 \pm 0.08[-28.91,-24.40]$ & $0.02 \pm 0.001-0.06 \pm 0.01$ \\
\hline & $\mathrm{CH}_{3} \mathrm{OH}\left(5_{0,5}-4_{0,4}\right)-\mathrm{A}^{+}$ & $-27.2 \pm 0.1$ & $1.47 \pm 0.13$ & $0.40 \pm 0.05$ & $0.60 \pm 0.08[-28.74,-25.07]$ & $0.02 \pm 0.01-0.08 \pm 0.01$ \\
\hline \multirow[t]{5}{*}{ IRAS 13037-6112 } & $\mathrm{SiO}(5-4)$ & $\ldots$ & $\ldots$ & $<0.12$ & & \\
\hline & ${ }^{13} \mathrm{CO}(2-1)$ & $-27.6 \pm 0.01$ & $2.64 \pm 0.04$ & $7.86 \pm 0.87(\mathrm{~B}) / 2.73 \pm 0.46(\mathrm{R})$ & $20.66 \pm 2.08[-30.34,-23.78]$ & \\
\hline & $\mathrm{C}^{17} \mathrm{O}(2-1)$ & $-27.1 \pm 0.02$ & $1.10 \pm 0.08$ & $1.91 \pm 0.27$ & $3.99 \pm 0.41[-30.30,-25.29]$ & $0.12 \pm 0.01$ \\
\hline & $\mathrm{CH}_{3} \mathrm{OH}\left(5_{-1,5}-4_{-1,4}\right)-\mathrm{E}$ & $-27.3 \pm 0.03$ & $1.15 \pm 0.09$ & $0.53 \pm 0.07$ & $0.73 \pm 0.08[-29.41,-24.73]$ & $0.03 \pm 0.01-0.11 \pm 0.01$ \\
\hline & $\mathrm{CH}_{3} \mathrm{OH}\left(5_{0,5}-4_{0,4}\right)-\mathrm{A}^{+}$ & $-27.6 \pm 0.04$ & $1.17 \pm 0.10$ & $0.43 \pm 0.06$ & $0.70 \pm 0.08[-29.41,-25.74]$ & $0.02 \pm 0.01-0.09 \pm 0.01$ \\
\hline SMM 5 & $\mathrm{C}^{17} \mathrm{O}(2-1)$ & $-26.4 \pm 0.04$ & $0.77 \pm 0.11$ & $0.81 \pm 0.16$ & $1.40 \pm 0.17[-28.59,-25.33]$ & $0.08 \pm 0.04$ \\
\hline SMM 6 & $\mathrm{C}^{17} \mathrm{O}(2-1)$ & $-27.0 \pm 0.03$ & $1.66 \pm 0.08$ & $1.56 \pm 0.20$ & $3.64 \pm 0.38[-30.01,-24.66]$ & $0.17 \pm 0.09$ \\
\hline SMM 7 & $\mathrm{C}^{17} \mathrm{O}(2-1)$ & $-26.3 \pm 0.04$ & $1.36 \pm 0.10$ & $1.21 \pm 0.16$ & $2.46 \pm 0.27[-28.96,-24.45]$ & $0.13 \pm 0.07$ \\
\hline IRAS 13039-6108 & $\mathrm{C}^{17} \mathrm{O}(2-1)$ & $-26.1 \pm 0.04$ & $1.14 \pm 0.07$ & $1.12 \pm 0.14$ & $1.92 \pm 0.21[-28.63,-24.66]$ & $0.07 \pm 0.01$ \\
\hline SMM 8 & $\mathrm{C}^{17} \mathrm{O}(2-1)$ & $-26.2 \pm 0.1$ & $1.42 \pm 0.33$ & $0.47 \pm 0.08$ & $1.28 \pm 0.18[-29.17,-24.33]$ & $0.05 \pm 0.02$ \\
\hline SMM 9 & $\mathrm{C}^{17} \mathrm{O}(2-1)$ & $-26.5 \pm 0.1$ & $2.01 \pm 0.46$ & $0.46 \pm 0.10$ & $1.22 \pm 0.19[-29.26,-25.24]$ & $0.05 \pm 0.02$ \\
\hline IRAS 13042-6105 & $\mathrm{C}^{17} \mathrm{O}(2-1)$ & $-26.5 \pm 0.1$ & $1.00 \pm 0.24$ & $0.40 \pm 0.07$ & $0.65 \pm 0.11[-28.21,-24.66]$ & $0.04 \pm 0.02$ \\
\hline
\end{tabular}

Notes. Columns (3)-(8) of this table are as follows: (3) LSR velocity; (4) FWHM linewidth; (5) peak line intensity; (6) integrated intensity; (7) peak optical thickness (see Sect. 4.3/Appendix A.3). ${ }^{(a)}$ For the ${ }^{13} \mathrm{CO}$ lines the intensities of the blue (B) and red (R) peak are given. ${ }^{(b)}$ Integrated intensity is derived by integrating over the velocity range indicated in square brackets. Note that the high-velocity blueshifted wing in the ${ }^{13} \mathrm{CO}$ spectrum towards SMM 2 was excluded from the integrated intensity. For DCN(3-2), the percentage $92.6 \%$ in brackets indicates the contribution of hf component's intensity lying within the detected line. ${ }^{(c)} \mathrm{A}$ blend of $\mathrm{CH}_{3} \mathrm{OH}\left(5_{4,1}-4_{4,0}\right)-\mathrm{A}^{+}$and $\mathrm{CH}_{3} \mathrm{OH}\left(5_{4,2}-4_{4,1}\right)-\mathrm{A}^{-}$.

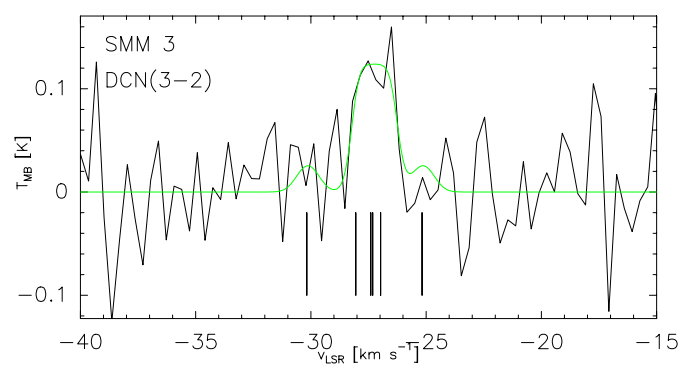

Fig. 6. The smoothed DCN(3-2) spectrum towards SMM 3. The line is overlaid with a hf-structure fit, and the vertical lines show the relative velocities of individual $\mathrm{hf}$ components.

$d=2.54 \pm 0.66 \mathrm{kpc}$. The corresponding Galactocentric distance is about $R_{\mathrm{GC}} \simeq 7.26 \mathrm{kpc}$ (see Appendix A.1 for further details).

\subsection{Revision of clump properties presented in Paper I}

We used the revised cloud distance $2.54 \pm 0.66 \mathrm{kpc}$ to recalculate the distance-dependent clump parameters presented in Paper I, where the value $d=2.4 \mathrm{kpc}$ was adopted. These parameters include the clump radius $(R \propto d)$, mass $\left(M \propto d^{2}\right)$, and volumeaveraged $\mathrm{H}_{2}$ number density $\left(\left\langle n\left(\mathrm{H}_{2}\right)\right\rangle \propto M / R^{3} \propto d^{-1}\right)$ (see Eqs. (6)-(8) in Paper I). The results are presented in Cols. (4)-(7) of Table 1 . The revised values are in the ranges $R \sim 0.3-0.5 \mathrm{pc}$, $M \sim 40-250 M_{\odot}, N\left(\mathrm{H}_{2}\right) \sim 1-3 \times 10^{22} \mathrm{~cm}^{-2}$, and $\left\langle n\left(\mathrm{H}_{2}\right)\right\rangle \sim$ $0.3-1 \times 10^{4} \mathrm{~cm}^{-3}$. For more details, we refer to Appendix A.2.
Figure 7 shows the clump masses as a function of effective radius. A least squares fit to the data gives $\log (M)=(3.08 \pm$ $0.23)+(2.82 \pm 0.55) \log (R)$, with the linear correlation coefficient $r=0.85$. For comparison, we also show the mass-radius threshold for massive-star formation, $M(R)=870 M_{\odot} \times(R / \mathrm{pc})^{1.33}$ inferred by Kauffmann \& Pillai (2010).

\subsection{Molecular column densities and fractional abundances}

Details on how the molecular column densities and fractional abundances were calculated are given in Appendix A.3. In brief, we used Eq. (A.3) to estimate the peak optical thickness for those lines, which were used in the column-density calculations (see Col. (7) of Table 3). In all cases, the lines appear to be optically thin $\left(\tau_{0} \ll 1\right)$. Therefore, the beam-averaged column densities of $\mathrm{C}^{17} \mathrm{O}, \mathrm{SiO}, \mathrm{CH}_{3} \mathrm{OH}$, and DCN were calculated using Eq. (A.4). The fractional abundances of the molecules were calculated by dividing the molecular column density by the $\mathrm{H}_{2}$ column density. For this purpose, the values of $N\left(\mathrm{H}_{2}\right)$ were derived from the LABOCA dust continuum map smoothed to the corresponding resolution of the line observations. The derived column densities and fractional abundances are listed in Table 4.

\subsection{CO depletion factors}

To estimate the amount of CO depletion in the clumps, we calculated the $\mathrm{CO}$ depletion factors, $f_{\mathrm{D}}$, following the analysis 
Table 4. Molecular column densities, fractional abundances with respect to $\mathrm{H}_{2}$, and CO depletion factors $\left(f_{\mathrm{D}}\right)$.

\begin{tabular}{lccccc}
\hline \hline Source & $\begin{array}{c}N\left(\mathrm{C}^{17} \mathrm{O}\right) \\
{\left[10^{14} \mathrm{~cm}^{-2}\right]}\end{array}$ & $\begin{array}{c}x\left(\mathrm{C}^{17} \mathrm{O}\right) \\
{\left[10^{-8}\right]}\end{array}$ & $f_{\mathrm{D}}$ & $\begin{array}{c}N\left(\mathrm{CH}_{3} \mathrm{OH}\right) \\
{\left[10^{13} \mathrm{~cm}^{-2}\right]}\end{array}$ & $\begin{array}{c}x\left(\mathrm{CH}_{3} \mathrm{OH}\right) \\
{\left[10^{-9}\right]}\end{array}$ \\
\hline SMM 1 & $4.7 \pm 1.3$ & $3.1 \pm 1.7$ & $2.2 \pm 1.2$ & $2.1 \pm 0.3-4.4 \pm 0.7$ & $1.3 \pm 0.8-2.7 \pm 1.6$ \\
SMM 2 & $3.4 \pm 1.0$ & $4.1 \pm 2.3$ & $1.7 \pm 0.9$ & $1.6 \pm 0.3-3.4 \pm 0.7$ & $0.2 \pm 0.1-0.4 \pm 0.1$ \\
SMM 3 & $4.9 \pm 1.4$ & $3.0 \pm 1.7$ & $2.3 \pm 1.3$ & $9.9 \pm 1.3-20.7 \pm 2.7$ & $5.8 \pm 3.3-12.2 \pm 6.9$ \\
SMM 4 & $15.1 \pm 3.9$ & $6.3 \pm 3.4$ & $1.1 \pm 0.6$ & $3.2 \pm 0.4-6.7 \pm 0.9$ & $1.3 \pm 0.7-2.8 \pm 1.5$ \\
IRAS 13037-6112 & $21.8 \pm 2.2$ & $21.9 \pm 12.1$ & $0.3 \pm 0.2$ & $3.8 \pm 0.4-7.9 \pm 0.9$ & $3.8 \pm 2.3-7.9 \pm 4.4$ \\
SMM 5 & $7.6 \pm 2.0$ & $13.0 \pm 7.2$ & $0.5 \pm 0.3$ & $\ldots$ & $\ldots$ \\
SMM 6 & $19.7 \pm 5.1$ & $12.2 \pm 6.7$ & $0.6 \pm 0.3$ & $\ldots$ & $\ldots$ \\
SMM 7 & $13.3 \pm 3.5$ & $16.5 \pm 9.1$ & $0.4 \pm 0.2$ & $\ldots$ & $\ldots$ \\
IRAS 13039-6108 & $10.5 \pm 1.1$ & $12.0 \pm 6.7$ & $0.6 \pm 0.3$ & $\ldots$ & $\ldots$ \\
SMM 8 & $6.9 \pm 1.9$ & $6.8 \pm 3.7$ & $1.0 \pm 0.5$ & $\ldots$ & $\ldots$ \\
SMM 9 & $6.6 \pm 1.9$ & $5.9 \pm 3.2$ & $1.2 \pm 0.6$ & $\cdots$ & $\ldots$ \\
IRAS 13042-6105 & $3.5 \pm 1.0$ & $5.1 \pm 2.8$ & $1.3 \pm 0.7$ & $\ldots$ & $\ldots$ \\
\hline
\end{tabular}

Notes. ${ }^{(a)}$ Towards SMM 3, we also determined the $\mathrm{SiO}$ and DCN column densities and abundances of $N(\mathrm{SiO})=6.5 \pm 1.2 \times 10^{11}-2.5 \pm 0.5 \times$ $10^{13} \mathrm{~cm}^{-2}, x(\mathrm{SiO})=4.0 \pm 2.4 \times 10^{-11}-1.5 \pm 0.9 \times 10^{-9}$, and $N(\mathrm{DCN})=6.7 \pm 1.8-48.7 \pm 12.8 \times 10^{10} \mathrm{~cm}^{-2}, x(\mathrm{DCN})=4.1 \pm 2.5-30.1 \pm 18.3 \times 10^{-12}$.

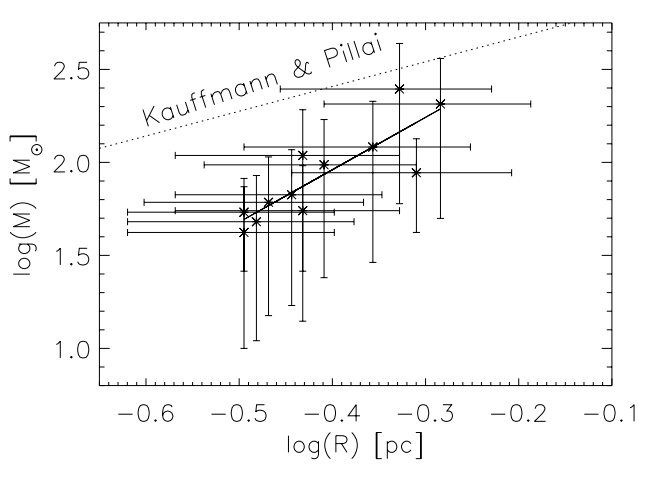

Fig. 7. Relation between mass and effective radius for the clumps in G304.74. The solid line shows the least squares fit to the data, i.e., $\log (M) \approx 3.1+2.8 \log (R)$. The dotted line represents the mass-radius threshold for massive-star formation proposed by Kauffmann \& Pillai (2010), i.e., $M(R)=870 M_{\odot} \times(R / p \mathrm{c})^{1.33}$.

outlined in Appendix A.4. The derived CO depletion factors, 0.3-2.3, are listed in Col. (4) of Table 4.

\subsection{Gas kinematics and internal pressure}

We used the measured $\mathrm{C}^{17} \mathrm{O}(2-1)$ linewidths to calculate $i$ ) the non-thermal portion of the line-of-sight velocity dispersion (averaged over a $277^{\prime \prime} 8$ beam), and ii) the level of internal turbulence (see below). The observed velocity dispersion is related to the FWHM linewidth as $\sigma_{\text {obs }}=\Delta v / \sqrt{8 \ln 2} \simeq \Delta v / 2.355$. The non-thermal velocity dispersion can then be calculated as (e.g., Myers et al. 1991)

$\sigma_{\mathrm{NT}}=\sqrt{\sigma_{\mathrm{obs}}^{2}-\frac{k_{\mathrm{B}} T_{\mathrm{kin}}}{m_{\mathrm{C}^{17} \mathrm{O}}}}$

where $m_{\mathrm{C}^{17} \mathrm{O}}=29 \mathrm{amu}$ is the mass of the $\mathrm{C}^{17} \mathrm{O}$ molecule. Furthermore, the level of internal turbulence is given by $f_{\text {turb }}=$ $\sigma_{\mathrm{NT}} / c_{\mathrm{s}}$, where $c_{\mathrm{s}}$ is the one-dimensional isothermal sound speed $\left(0.23 \mathrm{~km} \mathrm{~s}^{-1}\right.$ in a $15 \mathrm{~K} \mathrm{H}_{2}$ gas with $10 \% \mathrm{He}$, where the mean molecular weight per free particle is 2.33). The values of $\sigma_{\mathrm{NT}}$ and $f_{\text {turb }}$ are given in Cols. (2) and (3) of Table 5, respectively. The errors in these parameters were derived by propagating the errors in $\Delta v$ and $T_{\text {kin. }}$. In Fig. 8, we plot the FWHM linewidths
Table 5. Non-thermal velocity dispersion $\left(\sigma_{\mathrm{NT}}\right)$, the level of internal turbulence $\left(f_{\text {turb }}\right)$, and the total internal kinetic pressure $\left(P_{\text {kin }}\right)^{a}$.

\begin{tabular}{lccc}
\hline \hline Source & $\begin{array}{c}\sigma_{\mathrm{NT}} \\
{\left[\mathrm{km} \mathrm{s}^{-1}\right]}\end{array}$ & $f_{\text {turb }}$ & $\begin{array}{c}P_{\text {kin }} / k_{\mathrm{B}} \\
{\left[10^{5} \mathrm{~K} \mathrm{~cm}^{-3}\right]}\end{array}$ \\
\hline SMM 1 & $0.28 \pm 0.06$ & $1.2 \pm 0.3$ & $2.6 \pm 2.0$ \\
SMM 2 & $0.24 \pm 0.08$ & $1.0 \pm 0.4$ & $2.2 \pm 1.7$ \\
SMM 3 & $0.35 \pm 0.15$ & $1.5 \pm 0.7$ & $3.5 \pm 3.2$ \\
SMM 4 & $0.51 \pm 0.06$ & $2.2 \pm 0.5$ & $9.7 \pm 7.3$ \\
IRAS 13037-6112 & $0.46 \pm 0.03$ & $1.6 \pm 0.1$ & $6.6 \pm 3.3$ \\
SMM 5 & $0.32 \pm 0.05$ & $1.4 \pm 0.3$ & $2.7 \pm 1.8$ \\
SMM 6 & $0.70 \pm 0.03$ & $3.0 \pm 0.5$ & $15.3 \pm 12.3$ \\
SMM 7 & $0.57 \pm 0.04$ & $2.5 \pm 0.5$ & $5.3 \pm 4.3$ \\
IRAS 13039-6108 & $0.47 \pm 0.03$ & $1.7 \pm 0.1$ & $2.5 \pm 1.7$ \\
SMM 8 & $0.60 \pm 0.14$ & $2.6 \pm 0.7$ & $8.2 \pm 6.7$ \\
SMM 9 & $0.85 \pm 0.20$ & $3.7 \pm 1.0$ & $17.6 \pm 15.2$ \\
IRAS 13042-6105 & $0.42 \pm 0.10$ & $1.8 \pm 0.5$ & $3.9 \pm 3.6$ \\
\hline
\end{tabular}

Notes. ${ }^{(a)}$ All the parameters were calculated assuming $T_{\text {kin }}=15 \pm 5 \mathrm{~K}$ for all the other sources except the IRAS sources 13037 and 13039 , for which we used the value $T_{\text {kin }}=22 \mathrm{~K}$.

as a function of clump effective radius (left panel), and the derived $f_{\text {turb }}$ values as a function of clump mass (right panel). The linewidths do not show any clear correlation with the clump size; they are, instead, scattered around a horizontal regression line (the fit is not shown), which suggests that the linewidths are more or less "constant" as a function of clump radius. As can be seen from the right panel of Fig. 8, the clumps are characterised by trans- to supersonic $\left(\sigma_{\mathrm{NT}} \gtrsim c_{\mathrm{s}}\right)$ non-thermal motions.

We also calculated the total (thermal+non-thermal) kinetic pressure within the clumps to be

$P_{\text {kin }}=P_{\mathrm{T}}+P_{\mathrm{NT}}=n k_{\mathrm{B}} T_{\text {kin }}+\rho \sigma_{\mathrm{NT}}^{2}=\rho\left(c_{\mathrm{s}}^{2}+\sigma_{\mathrm{NT}}^{2}\right)$,

where $\rho=2.33 m_{\mathrm{H}}\left\langle n\left(\mathrm{H}_{2}\right)\right\rangle$ is the average gas-mass density of the clump, and $m_{\mathrm{H}}$ is the mass of a hydrogen atom. The results, $\sim 2-18 \times 10^{5} \mathrm{~K} \mathrm{~cm}^{-3}\left(6.7 \times 10^{5} \mathrm{~K} \mathrm{~cm}^{-3}\right.$ on average $)$, are listed in Col. (4) of Table 5. We note that the pressures have considerable associated errors because of the assumed $\pm 5 \mathrm{~K}$ uncertainty in temperature.

\subsection{Virial analysis}

To examine the dynamical state of the clumps, we calculated their virial masses by ignoring the effects of external pressure, 

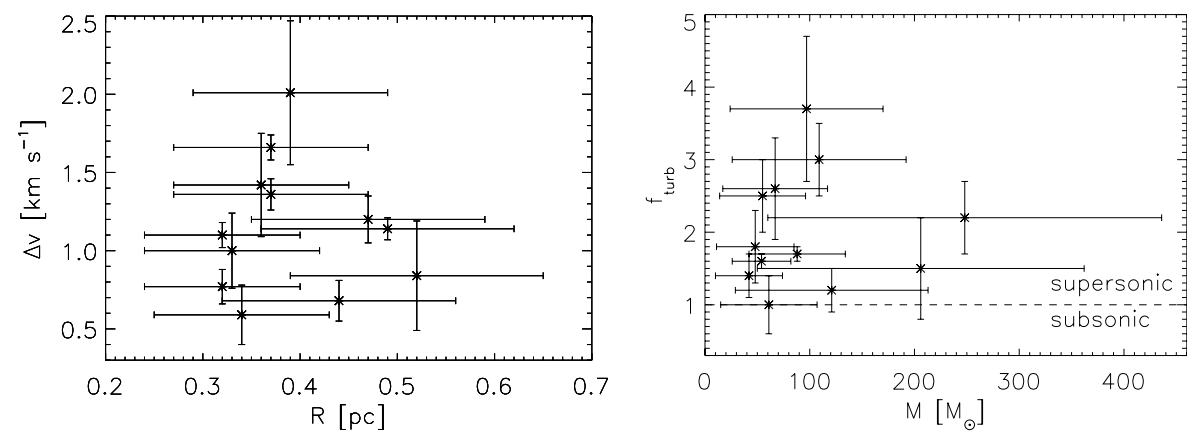

Fig. 8. Left: the FWHM linewidth of $\mathrm{C}^{17} \mathrm{O}(2-1)$ versus clump effective radius. Right: the $\sigma_{\mathrm{NT}} / c_{\mathrm{s}}$ ratio versus clump mass. The dashed line represents the limit between subsonic and supersonic non-thermal motions, i.e., $\sigma_{\mathrm{NT}} / c_{\mathrm{s}}=1$.

Table 6. The clump virial masses and virial parameters, and the estimated external pressure required to bring the clump into virial equilibrium.

\begin{tabular}{lccc}
\hline \hline Source & $\begin{array}{c}M_{\text {vir }} \\
{\left[M_{\odot}\right]}\end{array}$ & $\alpha_{\text {vir }}$ & $\begin{array}{c}P_{\text {ext }}^{\text {eq }} / k_{\mathrm{B}} \\
{\left[10^{5} \mathrm{~K} \mathrm{~cm}^{-3}\right]}\end{array}$ \\
\hline SMM 1 & $54 \pm 20$ & $0.4 \pm 0.4$ & $-2.5^{a}$ \\
SMM 2 & $35 \pm 16$ & $0.6 \pm 0.5$ & $-1.4^{a}$ \\
SMM 3 & $84 \pm 54$ & $0.4 \pm 0.4$ & $-4.1^{a}$ \\
SMM 4 & $132 \pm 44$ & $0.5 \pm 0.4$ & $-6.8^{a}$ \\
IRAS 13037-6112 & $85 \pm 23$ & $1.6 \pm 0.9$ & 3.0 \\
SMM 5 & $46 \pm 15$ & $1.1 \pm 0.9$ & 0.5 \\
SMM 6 & $183 \pm 52$ & $1.7 \pm 1.4$ & 7.0 \\
SMM 7 & $128 \pm 39$ & $2.3 \pm 1.9$ & 3.2 \\
IRAS 13039-6108 & $137 \pm 39$ & $1.6 \pm 0.9$ & 0.7 \\
SMM 8 & $135 \pm 64$ & $2.0 \pm 1.8$ & 4.7 \\
SMM 9 & $273 \pm 136$ & $2.8 \pm 2.5$ & 12.3 \\
IRAS 13042-6105 & $69 \pm 32$ & $1.4 \pm 1.3$ & 1.4 \\
\hline
\end{tabular}

Notes. ${ }^{(a)}$ The negative values suggest that stronger/additional internal pressure is needed to bring the clump into virial balance (see text).

rotation, and magnetic field in using the formula

$M_{\mathrm{vir}}=\frac{5}{8 \ln 2} \frac{R \Delta v_{\mathrm{ave}}^{2}}{a G}$,

where $\Delta v_{\text {ave }}$ is the width of the spectral line emitted by the molecule of mean mass $\mu=2.33$, and $G$ is the gravitational constant. The parameter $a=(1-p / 3) /(1-2 p / 5)$, where $p$ is the power-law index of the density profile $\left[n(r) \propto r^{-p}\right]$, is a correction for deviations from uniform density (Bertoldi \& McKee 1992, hereafter, BM92, their Appendix A). In the clump's gravitational potential energy, the $a$ parameter appears as

$\mathcal{W}=-\frac{3}{5} a \frac{G M^{2}}{R}$.

We note that the effect of the clump's ellipticity is neglected here (see BM92). We adopted the value $p=1.6(a=1.3)$ found by Beuther et al. (2002) for a sample of high-mass star-forming clumps. As a function of the observed linewidth, $\Delta v_{\text {obs }}, \Delta v_{\text {ave }}$ is given by (Fuller \& Myers 1992)

$\Delta v_{\mathrm{ave}}^{2}=\Delta v_{\mathrm{T}}^{2}+\Delta v_{\mathrm{NT}}^{2}=\Delta v_{\mathrm{obs}}^{2}+8 \ln 2 \times \frac{k_{\mathrm{B}} T_{\mathrm{kin}}}{m_{\mathrm{H}}}\left(\frac{1}{\mu}-\frac{1}{\mu_{\mathrm{mol}}}\right)$,

where $\Delta v_{\mathrm{T}}$ is the thermal linewidth, $\mu=2.33$, and $\mu_{\mathrm{mol}}=29$ for $\mathrm{C}^{17} \mathrm{O}$, which is the molecule that we employed in the analysis. The virial masses are listed in Col. (2) of Table 6. The associated error was propagated from those of $R, \Delta v_{\mathrm{obs}}$, and $T_{\text {kin }}$.

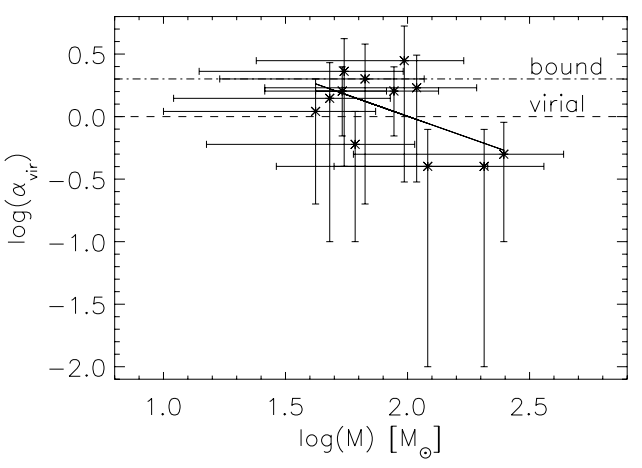

Fig. 9. Relation between virial parameter and mass for the clumps in G304.74. The solid line is the least squares fit to the data, i.e., $\log \left(\alpha_{\text {vir }}\right) \simeq$ 1.4-0.7 $\log (M)$. The dashed line indicates the virial-equilibrium limit of $\alpha_{\mathrm{vir}}=1$, and the dash-dotted line shows the limit of gravitational boundedness or $\alpha_{\text {vir }}=2$.

The virial parameters of the clumps were calculated following the definition by BM92, i.e.,

$\alpha_{\mathrm{vir}}=\frac{2 \mathcal{T}}{|\mathcal{W}|}=\frac{M_{\mathrm{vir}}}{M}$,

where $\mathcal{T}$ is the internal kinetic energy of the clump. The value $\alpha_{\text {vir }}=1$ corresponds to the virial equilibrium, i.e., $2\langle\mathcal{T}\rangle+\langle\mathcal{W}\rangle=$ 0 . The value $\alpha_{\text {vir }} \leq 2$ corresponds to the self-gravitating limit defined by $\langle\mathcal{T}\rangle+\langle\mathcal{W}\rangle \leq 0$ (e.g., Pound \& Blitz 1993) $)^{2}$. The derived $\alpha_{\text {vir }}$ values are given in Col. (3) of Table 6. The uncertainty was derived by propagating the errors in both mass estimates.

The derived $\alpha_{\text {vir }}$ values, $\sim 0.4-2.8$, are plotted as a function of the clump mass in Fig. 9. Although the $\alpha_{\text {vir }}$ values are associated with considerable uncertainties, most of the clumps appear to be gravitationally bound, and some of them are close virial equilibrium. Moreover, there is a hint of a negative correlation between $\alpha_{\text {vir }}$ and $M$. A least squares fit to the data points yields $\log \left(\alpha_{\text {vir }}\right)=(1.38 \pm 0.63)-(0.69 \pm 0.32) \log (M)$, with the correlation coefficient $r=-0.56$.

\subsection{The confining effect of external pressure}

The slope of the $\alpha_{\text {vir }}-M$ relation found above, -0.69 , is quite close to the value $-2 / 3 \simeq-0.67$ that is characteristic of pressureconfined clumps (BM92). Therefore, the external pressure may

2 In this case, it could be more appropriate to use the term "equipartition" parameter instead of "virial" parameter (Ballesteros-Paredes 2006; Heitsch et al. 2008). 
play an important role in the dynamics of the clumps. Taking the kinetic energy resulting from the surface pressure on the clump, $\mathcal{T}_{\text {ext }}$, into account, we can write the virial theorem in the form (e.g., McKee \& Zweibel 1992) ${ }^{3}$

$2\left(\langle\mathcal{T}\rangle-\left\langle\mathcal{T}_{\text {ext }}\right\rangle\right)+\langle\mathcal{W}\rangle=0$

The above energies can be written as a function of pressure as

$\mathcal{T}=\frac{3}{2} P_{\text {kin }} V_{\text {cl }}$,
$\mathcal{T}_{\text {ext }}=\frac{3}{2} P_{\text {ext }} V_{\text {cl }}$,

and

$\mathcal{W}=-3 P_{\text {grav }} V_{\mathrm{cl}}$

In the above formulae, $V_{\mathrm{cl}}=\frac{4}{3} \pi R^{3}$ is the clump volume, $P_{\mathrm{ext}}$ is the external pressure on the clump, and $P_{\text {grav }}$ is the gravitational pressure. We can write $P_{\text {grav }}$ as

$P_{\text {grav }}=-\frac{1}{3} \frac{W}{V_{\mathrm{cl}}}=-\frac{1}{3}\left(-\frac{3}{5} a \frac{G M^{2}}{R}\right) \frac{1}{\frac{4}{3} \pi R^{3}}=\frac{3 a}{20 \pi} \frac{G M^{2}}{R^{4}}$.

Substituting Eqs. (8)-(10) into Eq. (7), we get

$P_{\text {kin }}-P_{\text {ext }}-P_{\text {grav }}=0$.

From Eq. (12), we can estimate the $P_{\text {ext }}$ required to bring the clumps into virial equilibrium, which is roughly suggested by Fig. 9. The estimated $P_{\text {ext }}^{\mathrm{eq}}$ values are listed in Col. (4) of Table 6. We note that the values are associated with very large errors, and we only give the central values in the table. The clumps SMM 1-4 in the southern filament are formally characterised by negative external pressures. Although there are large uncertainties in the analysis, these clumps should be either warmer or have some additional internal pressure acting outward, such as magnetic pressure, in order to achieve virial equlibrium. The positive values of $P_{\mathrm{ext}}^{\mathrm{eq}}$ are found for the clumps north of SMM 4, to lie in the range $\sim 0.5-12 \times 10^{5} \mathrm{~K} \mathrm{~cm}^{-3}\left(\sim 3.3 \times 10^{5} \mathrm{~K} \mathrm{~cm}^{-3}\right.$ on average $)$.

\subsection{Mass infall}

The detected ${ }^{13} \mathrm{CO}(2-1)$ lines, most notably towards SMM 2, show the "classic" signature of the infalling gas motions, i.e., redshifted absorption dips, or blue asymmetric profiles (e.g., Lucas 1976; Myers et al. 1996). The optically thin $C^{17}$ O lines peak close to the radial velocity of the self-absorption dip. Therefore, we can presume that the double-peak profiles are not caused by two distinct velocity components along the line of sight. We note, however, that many of the absorption features are slightly redshifted with respect to the systemic velocity (Fig. 3). The blue asymmetry is weakest towards SMM 1, which indicates

\footnotetext{
${ }^{3}$ If the magnetic energy, $\mathcal{M}$, is also taken into account, the virial theorem is $2\left(\langle\mathcal{T}\rangle-\left\langle\mathcal{T}_{\text {ext }}\right\rangle\right)+\langle\mathcal{M}\rangle+\langle\mathcal{W}\rangle=0$. The only expansionary terms here are $\mathcal{T}$ and $\mathcal{M}$ (the angle brackets denoting time averages have been dropped for simplicity). Therefore, from the condition $\mathcal{M} \ll 2 \mathcal{T}$, we can estimate the upper limit to the magnetic field strength, $B$, when Eq. (7) is still expected to be valid. If we neglect any contribution from the magnetic field outside the clump, and assume that the field inside the clump is uniform, we can write $\mathcal{M}=B^{2} V_{\mathrm{cl}} / 2 \mu_{0}$, where $\mu_{0}$ is the vacuum permeability. This leads to an upper limit of $B \ll \sqrt{4 \mu_{0} \mathcal{T} / V_{\mathrm{cl}}}=\sqrt{6 \mu_{0} P_{\mathrm{kin}}}$.
}

Table 7. Mass-infall parameters.

\begin{tabular}{lcccc}
\hline \hline Source & $\begin{array}{c}V_{\text {inf }} \\
{\left[\mathrm{km} \mathrm{s}^{-1}\right]}\end{array}$ & $\begin{array}{c}\dot{M}_{\text {inf }} \\
{\left[10^{-5} M_{\odot} \mathrm{yr}^{-1}\right]}\end{array}$ & $\begin{array}{c}\tau_{\text {inf }} \\
{\left[10^{5} \mathrm{yr}\right]}\end{array}$ & $V_{\text {inf }} / V_{\text {ff }}$ \\
\hline SMM 1 & $0.03 \pm 0.01$ & $2.6 \pm 1.0$ & $45.8 \pm 17.4$ & $0.02 \pm 0.01$ \\
SMM 2 & $0.03 \pm 0.02$ & $1.8 \pm 1.2$ & $33.1 \pm 21.4$ & $0.02 \pm 0.02$ \\
SMM 3 & $0.17 \pm 0.14$ & $23.7 \pm 19.6$ & $8.7 \pm 7.2$ & $0.09 \pm 0.08$ \\
SMM 4 & $0.20 \pm 0.05$ & $36.1 \pm 9.0$ & $6.9 \pm 1.7$ & $0.09 \pm 0.04$ \\
IRAS 13037 & $0.16 \pm 0.02$ & $9.8 \pm 1.2$ & $5.5 \pm 0.7$ & $0.13 \pm 0.04$ \\
\hline
\end{tabular}

that in this clump there is the slowest infalling gas. We estimated the infall velocity, $V_{\text {inf }}$, using Eq. (9) of Myers et al. (1996)

$V_{\mathrm{inf}} \approx \frac{\sigma^{2}}{v_{\text {red }}-v_{\text {blue }}} \ln \left(\frac{1+e \times T_{\mathrm{BD}} / T_{\mathrm{dip}}}{1+e \times T_{\mathrm{RD}} / T_{\mathrm{dip}}}\right)$,

where $\sigma$ is the velocity dispersion derived from the appropriate optically thin line (we used the $\mathrm{C}^{17} \mathrm{O}$ lines), $v_{\text {red }}-v_{\text {blue }}$ is the difference between the radial velocities of the red and blue peaks, $T_{\mathrm{BD}}$ and $T_{\mathrm{RD}}$ are the heights of the blue and red peaks above the absorption dip, and $T_{\text {dip }}$ is the intensity of the absorption dip (see also Fig. 2 of Myers et al. 1996, for the definitions). The obtained values, $\sim 0.03-0.20 \mathrm{~km} \mathrm{~s}^{-1}$, are listed in Col. (2) of Table 7 . The uncertainty in $V_{\text {inf }}$ was estimated from the error in the FWHM linewidth of $\mathrm{C}^{17} \mathrm{O}(2-1)$.

Assuming spherically symmetric infall, and that the mass contained in the spherical shell is $\mathrm{d} M(r)=4 \pi r^{2} \rho \mathrm{d} r$, the infall velocities can be used to estimate the mass infall rate as

$\dot{M}_{\mathrm{inf}}=\frac{\mathrm{d} M}{\mathrm{~d} t}=\frac{\mathrm{d} M}{\mathrm{~d} r} \frac{\mathrm{d} r}{\mathrm{~d} t}=4 \pi r_{\mathrm{inf}}^{2} \rho V_{\mathrm{inf}}$,

where $r_{\text {inf }}$ is the size of the infalling region (taken to be the clump effective radius), and $\rho=2.33 m_{\mathrm{H}}\left\langle n\left(\mathrm{H}_{2}\right)\right\rangle$ is again the average gas mass density ${ }^{4}$. The derived values, $\sim 2-36 \times 10^{-5} M_{\odot} \mathrm{yr}^{-1}$, with uncertainties estimated from the errors in $V_{\text {inf }}$, are given in Col. (3) of Table 7.

Assuming that $\dot{M}_{\text {inf }}$ remains constant, the infall timescale can be calculated from

$\tau_{\text {inf }}=\frac{M}{\dot{M}_{\text {inf }}}$.

The obtained timescales, $\sim 6-46 \times 10^{5} \mathrm{yr}$, are listed in Col. (4) of Table 7 (the uncertainty is based on that in $\dot{M}_{\text {inf }}$ ).

We also calculated the free-fall velocities of the clumps

$V_{\mathrm{ff}}=\sqrt{\frac{2 G M}{R}}$

which were then compared with the derived infall velocities. In the last column of Table 7 , we list the $V_{\text {inf }} / V_{\mathrm{ff}}$ ratios. The associated uncertainty was propagated from those of $V_{\mathrm{inf}}, M$, and $R$. In all cases, the infall velocity is much lower than the corresponding free-fall velocity.

\subsection{Fragmentation and stability analysis of the filament}

The IRDC G304.74 is highly filamentary in shape, its total mass being about $\sim 1200 M_{\odot}$ (the sum of all clump masses), and it is resolved into twelve submm clumps at $\sim 20^{\prime \prime}$ resolution. The

${ }^{4}$ We note, however, that the density of the absorbing and infalling gas may be somewhat lower than the volume-average clump density. 
projected linear extent of filament's long axis is about $12^{\prime}$ or $8.9 \mathrm{pc}$, and the filament's mass per unit length, or line mass, is $M_{\text {line }} \simeq 135 M_{\odot} \mathrm{pc}^{-1}$. We note that this line mass may only represent a lower limit, because there is likely to be a lot of mass on larger scales (Kainulainen et al. 2011b). The projected separations between the clump submm peak positions lie in the range $22^{\prime \prime}-115^{\prime \prime}$, or $0.27-1.42 \mathrm{pc}$ at the cloud distance $\left(61^{\prime \prime}=0.75 \mathrm{pc}\right.$ on average). The shortest separations are found near SMM 6 and IRAS 13037, whereas the separations are largest around SMM 7.

To examine the fragmentation of G304.74 in more detail, we first calculate its thermal Jeans length from

$\lambda_{\mathrm{J}}=\frac{c_{\mathrm{s}}^{2}}{G \Sigma_{0}}$,

where $c_{\mathrm{S}}$ was adopted to be $0.23 \mathrm{~km} \mathrm{~s}^{-1}$ (Sect. 4.5), $\Sigma_{0}=$ $2.33 m_{\mathrm{H}} N\left(\mathrm{H}_{2}\right)$ is the surface density, and $N\left(\mathrm{H}_{2}\right)$ refers to the central column density for which we use the median value $\sim 1.4 \times 10^{22} \mathrm{~cm}^{-2}$ (see Col. (6) of Table 1). The resulting thermal Jeans length is only $\sim 0.05 \mathrm{pc}$, which is about five times less than the spatial resolution of our LABOCA map, and therefore much shorter than the observed minimum clump separation. Thermal Jeans instability does not appear to be responsible for the cloud fragmentation on the scale of clumps.

On the other hand, we found that the filament is dominated by supersonic non-thermal motions. Therefore, it may be appropriate to replace $c_{\mathrm{s}}$ in Eq. (17) with the effective sound speed, which takes both thermal and non-thermal motions into account, i.e., $c_{\mathrm{eff}}=\left(c_{\mathrm{s}}^{2}+1 / 3 \times \sigma_{\mathrm{NT}, 3 \mathrm{D}}^{2}\right)^{1 / 2}$, where $\sigma_{\mathrm{NT}, 3 \mathrm{D}}$ is the three-dimensional non-thermal velocity dispersion (e.g., Chandrasekhar 1951; Bonazzola et al. 1987). Here we have assumed isotropic non-thermal motions, i.e., $\sigma_{\mathrm{NT}, 3 \mathrm{D}}=\sqrt{3} \sigma_{\mathrm{NT}, 1 \mathrm{D}}$. Using the median value of $\sigma_{\mathrm{NT}, 1 \mathrm{D}}=0.47 \mathrm{~km} \mathrm{~s}^{-1}$ (see Col. (2) of Table 5), the effective, or "turbulent" Jeans length becomes $\sim 0.24 \mathrm{pc}$. This is about five times larger than the thermal Jeans length, but still much smaller than the average clump separation of $\sim 0.75 \mathrm{pc}$. Therefore, the observed fragmentation of the filament cannot be attributed either to non-thermal Jeans-type instability.

According to the magnetohydrodynamic "sausage"-instability theory, fragmentation of a self-gravitating fluid cylinder will lead to the formation of distinct condensations with almost periodic separations. This fragmentation length-scale corresponds to the wavelength at which the instability grows the fastest (e.g., Chandrasekhar \& Fermi 1953; Nagasawa 1987; see also Jackson et al. 2010). If the cylinder's radius is $R_{\text {cyl }}$, then, for an incompressible fluid, perturbation analysis shows that the above critical wavelength is $\lambda_{\max }^{\text {incomp. }} \simeq 11 R_{\text {cyl }}$. If we use as $R_{\text {cyl }}$ the mean clump effective radius of $\sim 0.4 \mathrm{pc}$, we obtain $\lambda_{\max }^{\text {incomp. }} \simeq 4.4 \mathrm{pc}$. This is clearly a longer length scale than observed in G304.74. On the other hand, for an isothermal, infinitely long gas cylinder the fastest growing mode appears at $\lambda_{\max }^{\text {isotherm. }} \simeq 22 H$, where $H=c_{\mathrm{s}} / \sqrt{4 \pi G \rho_{0}}$ is the isothermal scale height with $\rho_{0}$ the central gas mass density along the axis of the cylinder (e.g., Nagasawa 1987). If we compute $\rho_{0}$ assuming that the central number density is $10^{5} \mathrm{~cm}^{-3}$, we obtain the scale height $H=0.013 \mathrm{pc}$, and thus $\lambda_{\max }^{\text {isotherm. }} \simeq 0.3 \mathrm{pc}$. This is comparable to the effective Jeans-length calculated above. If the value of $H$ is calculated using the effective sound speed, $c_{\text {eff }}$, i.e., we calculate the effective scale height, we get $H_{\mathrm{eff}}=0.03 \mathrm{pc}$, and $\lambda_{\max }^{\text {isotherm. }} \simeq 0.7 \mathrm{pc}$. This wavelength is close to the observed average clump separation. We also note that it is comparable to the average width of the filament, $\sim 0.8 \mathrm{pc}$, in accordance with the theory (Nakamura et al. 1993).
We next examine whether the G304.74 filament could be susceptible to axisymmetric perturbations or radial collapse. For an unmagnetised, infinite, isothermal filament, the instability is reached if its $M_{\text {line }}$ value exceeds the critical equilibrium value of $M_{\text {line }}^{\text {crit }}=2 c_{\mathrm{s}}^{2} / G$ (e.g., Ostriker 1964; Inutsuka \& Miyama 1992). At $T_{\text {kin }}=15 \mathrm{~K}$, the critical value is $M_{\text {line }}^{\text {crit }} \simeq 25 M_{\odot} \mathrm{pc}^{-1}$. Using this value, we estimate that the ratio $M_{\text {line }} / M_{\text {line }}^{\text {crit }} \simeq 5$ for G304.74. Thus, G304.74 appears to be a thermally supercritical filament susceptible to fragmentation, in agreement with the detected clumpy structure. If the external pressure is not negligible, as may be the case for G304.74, then $M_{\text {line }}^{\text {crit }}$ is actually smaller than the above value (e.g., Fiege \& Pudritz 2000a), making the filament even more supercritical. However, owing to the presence of non-thermal motions, it may be appropriate to replace $M_{\text {line }}^{\text {crit }}$ with the virial mass per unit length, $M_{\text {line }}^{\text {vir }}=2\left\langle\sigma^{2}\right\rangle / G$, where $\left\langle\sigma^{2}\right\rangle$ is the square of the total velocity dispersion obtained from Eq. (5) as $\left\langle\sigma^{2}\right\rangle=\Delta v_{\text {ave }}^{2} / 8 \ln 2$ (Fiege \& Pudritz 2000a). Using again the value $T_{\text {kin }}=15 \mathrm{~K}$ and the $\mathrm{C}^{17} \mathrm{O}$ linewidths, we obtain the mean velocity dispersion of $\left\langle\sigma^{2}\right\rangle^{1 / 2}=0.54 \mathrm{~km} \mathrm{~s}^{-1}$. This leads to the values $M_{\text {line }}^{\text {vir }} \simeq 136 M_{\odot} \mathrm{pc}^{-1}$ and $M_{\text {line }} / M_{\text {line }}^{\text {vir }} \simeq 1$. In this case, G304.74 as a whole would be near virial equilibrium. The fragmentation timescale for a filament of radius $R_{\mathrm{cyl}}$ is expected to be comparable to its radial signal crossing time, $\tau_{\text {cross }}=R_{\text {cyl }} /\left\langle\sigma^{2}\right\rangle^{1 / 2}$ (see Eq. (26) in Fiege \& Pudritz 2000b), which for G304.74 is estimated to be $\sim 7.2 \times 10^{5} \mathrm{yr}$. We note that the value of $\left\langle\sigma^{2}\right\rangle^{1 / 2}$, and thus $M_{\text {line }}^{\text {vir }}$, may increase if the cloud's gravitational potential energy is converted into gas kinetic energy during the contraction of the cloud. We also note that in the case of a magnetised molecular-cloud filament, the critical mass per unit length is unlikely to differ from that of an unmagnetised filament by more than a factor of order unity (Fiege \& Pudritz 2000a,b).

\section{Discussion}

\subsection{Fragmentation and dynamical state of G304.74+01.32}

The average projected clump separation in G304.74 is about $0.75 \mathrm{pc}$. As shown above, this value can be explained if the fragmentation of the parent filament is caused by a "sausage"-type (or pinch) instability, where the dominant unstable mode appears at the wavelength depending on the effective sound speed. This instability might have been triggered by, e.g., an increase in the radial inward force or a radial contraction leading to an increase in the tension force of the azimuthal magnetic field. This conforms to the results of Jackson et al. (2010), who speculated that the "sausage"-type instability is the main physical driver of the fragmentation of filamentary IRDCs. Henning et al. (2010) and Kang et al. (2011) found that the mean clump separation is $0.9 \mathrm{pc}$ in both the filamentary IRDC G011.11-0.12 or the Snake, and IRDC G049.40-00.01, respectively. This resembles the situation in G304.74, and could therefore be indicative of a similar fragmentation process. In Paper I, we discussed the possibility that the origin of the G304.74 filament, and its fragmentation into clumps is caused by supersonic turbulent flows. Even though the formation of the cloud might have been caused by converging turbulent flows, the role of random turbulence in fragmenting the filament appears unclear in the light of the present results. Nevertheless, our new results here support the importance of non-thermal motions in G304.74.

The slope of the $\alpha_{\mathrm{vir}}-M$ relation that we found is roughly consistent with the prediction of BM92 for clumps confined by ambient pressure from their surrounding medium, i.e., $\alpha_{\text {vir }} \propto M^{-2 / 3}$. This slope can be understood as follows. On the 
one hand, the clump mass was found to be roughly proportional to $R^{3}$ (Fig. 7). On the other hand, the linewidth-radius "relation" shown in Fig. 8 is roughly flat, i.e., $\Delta v \approx$ const. Therefore, from Eqs. (3) and (6) we see that $\alpha_{\text {vir }} \propto R \Delta v^{2} / M \propto M^{1 / 3} M^{-1} \propto$ $M^{-2 / 3}$. Although our clumps, or most of them, appear to be gravitationally bound ( $\left.\alpha_{\text {vir }} \leq 2\right)$, it is possible that the ambient pressure (still) plays a non-negligible role in the clump dynamics. We note that for real pressure-confined clumps, $\alpha_{\text {vir }} \gg 1$, and the clumps are not gravitationally bound (BM92).

Could the external pressure be caused by turbulent flow motions outside the cloud? To estimate the turbulent ram pressure, $P_{\text {ram }}=\rho \sigma_{\mathrm{NT}}^{2}$, we use the average ${ }^{13} \mathrm{CO}(2-1)$ linewidth of $\sim 2.2 \mathrm{~km} \mathrm{~s}^{-1}$ derived for five of our clumps, and assume the density of the ${ }^{13} \mathrm{CO}$ emitting gas to be $10^{3} \mathrm{~cm}^{-3}$ (following Lada et al. 2008). If $T_{\text {kin }}$ is in the range 10-20 K, the corresponding non-thermal velocity dispersion is $\sigma_{\mathrm{NT}} \simeq 0.93 \mathrm{~km} \mathrm{~s}^{-1}$, which yields the value $P_{\text {ram }} / k_{\mathrm{B}} \sim 3 \times 10^{5} \mathrm{~K} \mathrm{~cm}^{-3}$. This is only about two times lower than the average internal kinetic pressure within the clumps, and very close to the average external pressure required for virial equilibrium $\left(P_{\mathrm{ram}} \simeq P_{\mathrm{ext}}^{\mathrm{eq}}\right.$; Sect. 4.7). Therefore, this supports the scenario of the importance of turbulence presented in Paper I. The obtained $P_{\text {ram }} / k_{\mathrm{B}}$ value is also consistent with intercloud pressures of $10^{4}-10^{6} \mathrm{~K} \mathrm{~cm}^{-3}$ deduced from theoretical estimates and different observations (see Field et al. 2011, and references therein). However, we note that the large-scale turbulent motions are anisotropic, and therefore that the associated pressure may be unable to confine the cloud, but could rather distort and/or even disrupt it (e.g., Ballesteros-Paredes 2006). The finding that $M_{\text {line }} / M_{\text {line }}^{\text {vir }} \simeq 1$ for the G304.74 filament suggests that it is close to virial equilibrium. The virial balance may be difficult to explain in the context of the turbulent fragmentation discussed in Paper I, because turbulent motions are expected to induce a flux of mass, momentum, and energy between the cloud and its surrounding medium (BallesterosParedes 2006). In addition, the detection of large-scale infall motions towards G304.74 suggests it to be out-of-equilibrium. For comparison, the Herschel study of the Aquila rift and Polaris Flare regions by André et al. (2010) showed that practically all filaments with $M_{\text {line }} / M_{\text {line }}^{\text {crit }}>1$ were associated with prestellar cores and/or embedded protostars. On the other hand, filaments with $M_{\text {line }} / M_{\text {line }}^{\text {crit }}<1$ were generally found to lack such starformation signatures. More observations of higher resolution would be needed to characterise more clearly the star-formation activity in G304.74, and compare it with the filamentary structures revealed by Herschel.

If the $M_{\text {line }} / M_{\text {line }}^{\text {vir }}$ ratio is indeed $\simeq 1$, then from Eq. (11) of Fiege \& Pudritz (2000a), and the average ratio of the external to internal pressure of $\left\langle P_{\text {ext }}^{\text {eq }}\right\rangle /\left\langle P_{\text {kin }}\right\rangle \simeq 0.49$, we estimate that the ratio of the total magnetic to kinetic energies per unit length is $\mathcal{M} /|\boldsymbol{W}| \simeq 0.49$. This value is $>0$, and therefore suggests that $i$ ) the overall effect of the magnetic field on the cloud is to provide support; and ii) the magnetic field is poloidally dominated. This disagrees with Fiege \& Pudritz (2000a), who found that the magnetic fields in the filamentary clouds that they analysed are likely to be helical and toroidally dominated. However, Fiege et al. (2004) analysed the Snake IRDC, which is more similar to G304.74, and concluded that it is likely dominated by the poloidal component of the magnetic field (or magnetically neutral), and therefore an excellent candidate for a magnetically supported filament. This may also be the case for the G304.74 filament. Interestingly, Fiege \& Pudritz (2000b) found that models with purely toroidal fields remain stable in "sausage" modes, whereas models with a poloidal field component may be unstable. We note, however, that the observed fragmentation of the filament strongly suggests that the magnetic field is not purely poloidal (or toroidal), because otherwise the fragmentation would have presumably been stabilised (Fiege \& Pudritz 2000b). If the filament fragmentation were caused by sausage-type instability, some toroidal magnetic field would probably needed, because the magnetic pressure associated with the poloidal field would resist the "squeezing" of the cloud.

On the other hand, the ambient pressure can also arise from the cloud's self-gravity. This was found to be the case for the Pipe Nebula by Lada et al. (2008), who found all the cores within the cloud to have $\alpha_{\text {vir }}>1$, and $\alpha_{\text {vir }} \propto M^{-0.66}$. The pressure due to the weight of the cloud is

$P_{\mathrm{cl}}=\frac{3 \pi}{20} G \Sigma^{2} \phi_{\mathrm{G}}$

where $\Sigma=M / \pi R^{2}$ is the mean mass surface density of the cloud, and $\phi_{\mathrm{G}}$ is a dimensionless correction factor apllied to account for the non-spherical geometry of a cloud (BM92; cf. our Eq. (11)). Following BM92, we use as the mean effective cloud radius the value $R \simeq \sqrt{0.8 \times 8.9 \mathrm{pc}^{2}} \simeq 2.7 \mathrm{pc}$, where $0.8 \mathrm{pc}$ is the average width of the filament. The corresponding surface density is $\Sigma \simeq 1 \mathrm{~g} \mathrm{~cm}^{-2}$. The aspect ratio of the filament is about $y \simeq 8.9 \mathrm{pc} / 0.8 \mathrm{pc} \sim 11$ (prolate), hence we adopt the value $\phi_{\mathrm{G}} \simeq 2$ (see Appendix B of BM92). Thus, we obtain the value $P_{\mathrm{cl}} \sim 5.5 \times 10^{4} \mathrm{~K} \mathrm{~cm}^{-3}$. This is about an order of magnitude lower than the average internal kinetic pressure within the clumps or the average $P_{\text {ext }}^{\mathrm{eq}}$ value, suggesting that the cloud's weight does not contribute significantly to the overall confining pressure. We note, however, that Kainulainen et al. (2011b) found that the masses of IRDCs traced by the thermal dust emission are generally only $\lesssim 10-20 \%$ of the masses traced by nearinfrared and $\mathrm{CO}$ data. Therefore, the pressure due to the weight of the cloud itself, which is mostly caused by the material at low column densities, is likely to be higher than estimated above.

Some of the clumps at the southern end of the filament were found to be characterised by negative external pressures in the case of virial equilibrium (or "energy equipartition"; Sect. 4.7), suggesting that they should be either warmer or have some additional internal pressure. Interestingly, this conforms to the results obtained in Paper I, where we used the dust opacities at $8 \mu \mathrm{m}$ and $870 \mu \mathrm{m}$ to estimate the dust temperatures towards the MIR-dark submm peaks. The clumps SMM 1 and 2 in the southern tip of the filament were estimated to have temperatures of $\sim 30 \mathrm{~K}$, but the values were associated with very large relative errors of $\sim 60-70 \%$ (thus we adopted $T_{\text {dust }}=15 \mathrm{~K}$ ). The masses (mean densities) of SMM 1 and 2 would be $\sim 44 M_{\odot}$ $\left(0.2 \times 10^{4} \mathrm{~cm}^{-3}\right)$ and $22 M_{\odot}\left(0.3 \times 10^{4} \mathrm{~cm}^{-3}\right)$, respectively, if we assumed that $T_{\text {dust }}=30 \mathrm{~K}$. The non-thermal velocity dispersions would be 0.27 and $0.23 \mathrm{~km} \mathrm{~s}^{-1}$, and the total kinetic pressures about $1.2 \times 10^{5} \mathrm{~K} \mathrm{~cm}^{-3}$ and $1.6 \times 10^{5} \mathrm{~K} \mathrm{~cm}^{-3}$, respectively. Consequently, the external pressures required to virial balance would become positive, i.e., $P_{\text {ext }}^{\text {eq }} / k_{\mathrm{B}} \sim 5.2 \times 10^{4}$ and $\sim 1.1 \times 10^{5} \mathrm{~K} \mathrm{~cm}^{-3}$, for SMM 1 and 2 , respectively. These are in reasonable agreement with the $P_{\mathrm{ext}}^{\mathrm{eq}}$ values estimated towards other clumps (see Col. (4) of Table 6). The present results seem to support the possibility of a north-to-south temperature gradient of $\sim 15 \rightarrow 30 \mathrm{~K}$ in G304.74.

\subsection{Clump kinematics, infall motions, and prospects for massive-star formation}

The clumps in G304.74 appear to be characterised by transonic to supersonic internal non-thermal motions. According to the 
turbulent core model of McKee \& Tan (2003), the clump (or core) has to be supersonically turbulent in order to achieve a high mass-accretion rate and be able to form massive stars. The presence of this turbulence is also expected to lead to the formation of substructure within the clump. Higher-resolution observations would be needed to find out whether the clumps have fragmented into smaller cores. For example, interferometric observations of a sample of clumps associated with IRDCs have revealed embedded cores within them (e.g., Rathborne et al. 2008; Hennemann et al. 2009; Wang et al. 2011).

The clump SMM 6, which has the second largest $f_{\text {turb }}$ value ( 3), is associated with two MSX point sources, suggesting that it indeed hosts subfragments, and that they have already been collapsed. On the other hand, as shown in Fig. 7, none of the clumps appear to clearly fullfil the mass-radius threshold for massive-star formation proposed by Kauffmann \& Pillai (2010). Only within the uncertainties could the two most massive clumps, SMM 3 and 4, exceed this limit.

The supersonic "turbulent" motions in the clumps may be due to gravitational infall motions (see Heitsch et al. 2009, and references therein). Evidence of large-scale infall motions in ${ }^{13} \mathrm{CO}$ lines was indeed found towards the southern clumps (see further discussion below). If the whole filament is in the process of hierarchical gravitational collapse with clumps collapsing locally, a complex velocity pattern is expected to be created. This "turbulence" is unable to resist gravitational collapse; it is instead generated from the collapse itself (Ballesteros-Paredes et al. 2011).

Radiative transfer models have shown that if the clump centre collapses faster than the outer layers, the observed spectral line should have two peaks with the blueshifted peak being stronger than the redshifted one (Zhou et al. 1993; Myers et al. 1996). The blue peak originates from the rear side of the clump, whereas the red peak comes from the front part of the clump. The central dip between the two peaks is caused by the envelope layer (see also Evans 1999). Similar line profiles have been seen towards SMM 1-4 and IRAS 13037, and are therefore likely undergoing large-scale inward motions. We derived the infall velocites and mass infall rates of $\sim 0.03-0.20 \mathrm{~km} \mathrm{~s}^{-1}$ and $\sim 2-36 \times$ $10^{-5} M_{\odot} \mathrm{yr}^{-1}$, respectively. These are comparable to the values $0.14 \mathrm{~km} \mathrm{~s}^{-1}$ and $3 \times 10^{-5} M_{\odot} \mathrm{yr}^{-1}$ found by Hennemann et al. (2009) towards J18364 SMM 1 South, which is a massive clump within an IRDC. Chen et al. (2010) measured higher infall velocities and rates of $\sim 0.7-4.6 \mathrm{~km} \mathrm{~s}^{-1}$ and $\sim 1-28 \times 10^{-3} M_{\odot} \mathrm{yr}^{-1}$, respectively, for their sample of MYSOs associated with extended green objects (EGOs) and IRDCs. Typically, the mass infall rates in MYSOs are $\sim 10^{-4}-10^{-2} M_{\odot} \mathrm{yr}^{-1}$ (e.g., Klaassen \& Wilson 2007; Zapata et al. 2008; Beltrán et al. 2011). We note that the infall timescales we derived, $\sim 6-46 \times 10^{5} \mathrm{yr}$, are quite long compared to the characteristic timescale of $\sim 10^{5} \mathrm{yr}$ for high-mass star formation (e.g., Davies et al. 2011, and references therein). The infall rate, however, may not remain constant during the star-formation process, which could also explain some of the large observed variations in the values quoted above.

We found that the infall velocities are one to two orders of magnitude lower than expectations for a free-fall collapse. This suggests that the large-scale collapse is not gravitationally dominated, but might be retarded by, e.g., magnetic fields. The predominantly poloidal magnetic field could resist motions perpendicular to the poloidal field lines, such as appears to be the case in the Snake IRDC (Fiege \& Pudritz 2000b; Fiege et al. 2004). We also emphasise that the derived infall parameters refer to the infall of the surrounding gas onto a (pre-)protocluster, not onto individual stars. For example, the timescale for (disk) accretion from the infalling envelope may be substantially shorter than the infall timescale. The current data do not allow us to decide whether the observed large-scale mass infall could feed competitive accretion in the clumps/protoclusters (Bonnell \& Bate 2006), or be related to monolithic collapse leading to massive star formation (McKee \& Tan 2003). On the one hand, the formation of high-mass stars in G304.74 seems uncertain according to the mass-radius threshold proposed by Kauffmann \& Pillai (2010). On the other hand, the high luminosites of the IRAS sources 13037 and 13039, 1.5-2 $\times 10^{3} L_{\odot}$ (Paper I), shows that at least intermediate-mass star- and/or cluster formation is taking place in this IRDC.

\subsection{The observed chemical properties of the clumps}

\subsubsection{CO depletion and the SiO detection towards SMM 3}

The CO depletion factors we derived are in the range $f_{\mathrm{D}} \sim$ 0.3-2.3. Therefore, $\mathrm{CO}$ molecules do not appear to be significantly depleted on the scale of clumps in G304.74, which conforms to the low average $\mathrm{H}_{2}$ densities. Higher-resolution observations would be needed to investigate whether the clumps contain smaller and more CO-depleted cores. The values $f_{\mathrm{D}}<1$ could be due to too small a canonical $\mathrm{CO}$ abundance being used, or contamination of non-depleted gas along the line of sight (see also Miettinen et al. 2011, and references therein). The latter possibility, however, may be less important because the cloud is located above the Galactic plane, and thus relatively free of contaminating emission along the line of sight. Uncertainties in $N\left(\mathrm{H}_{2}\right)$, particularly due to the dust opacity, propagate of course into those of $x\left(\mathrm{C}^{17} \mathrm{O}\right)$ and $f_{\mathrm{D}}$.

It is unsurprising that we found no evidence of $\mathrm{CO}$ freezeout towards the IRAS sources and SMM 6, which are all MIR bright and very likely associated with ongoing star formation. The physical processes associated with star-formation activity, such as outflows, heating, etc., are expected to release $\mathrm{CO}$ from the icy grain mantles back into the gas phase. The largest $f_{\mathrm{D}}$ values are seen consistently towards the MIR-dark clumps SMM 1 and 3. However, the detection of SiO emission in SMM 3 implies that there are outflows, because gas-phase $\mathrm{SiO}$ is expected to be produced when shocks disrupt the dust grains (e.g., Hartquist et al. 1980; Schilke et al. 1997). This agrees with the finding of Sakai et al. (2010, henceforth, SSH10), i.e., that the fraction of shocked gas (as traced by $\mathrm{SiO}$ ) is higher in the MSX-dark sources than in the MSX-bright sources. This could indicate that the MIR-dark clumps are associated with newly formed shocks giving rise to stronger $\mathrm{SiO}$ emission, whereas the weaker emission from the MIR-bright clumps could be due to shocks being older, and thus milder. We note that the highest estimated $\mathrm{SiO}$ column density value in SMM $3, \sim 2.5 \times 10^{13} \mathrm{~cm}^{-2}$, is comparable to the values $\sim 1.1-2.6 \times 10^{13} \mathrm{~cm}^{-2}$ found by SSH10 towards their MSX-dark clumps.

In contrast, Hernandez et al. (2011) found depletion factors of up to $f_{\mathrm{D}} \sim 5$ in the highly filamentary IRDC G035.39-00.33. Miettinen et al. (2011) derived the values $f_{\mathrm{D}} \sim 0.6-2.7$ towards a sample of clumps within different IRDCs, which are very similar to those obtained in the present work with the same telescope/angular resolution. Higher values of $f_{\mathrm{D}} \sim 6-19$ were found by Chen et al. (2011) for their sample of high-mass star-forming clumps, including sources within IRDCs, using the 10-m SMT observations of $\mathrm{C}^{18} \mathrm{O}$ at $34^{\prime \prime}$ resolution. This shows that strong $\mathrm{CO}$ depletion is possible even on the scale of clumps. We note that Hernandez et al. (2011) used the CO depletion timescale to constrain the lifetime of G035.39-00.33 (or the part 
of the cloud showing the strongest depletion). These investigations are useful considering the cloud formation timescales and mechanisms. Unfortunately, since we found no clear evidence of $\mathrm{CO}$ depletion in G304.74, we were unable to perform similar analysis.

\subsection{2. $\mathrm{CH}_{3} \mathrm{OH}$}

The fractional $\mathrm{CH}_{3} \mathrm{OH}$ abundances in the observed clumps were estimated to lie in the range $\sim 0.2-12 \times 10^{-9}$. The lowest values are found towards the MIR-dark clump SMM 2, and the highest ones towards SMM 3 and IRAS 13037.

The pure gas-phase chemical models suggest that $\mathrm{CH}_{3} \mathrm{OH}$ is formed from methylium cation through the radiative association reaction $\mathrm{CH}_{3}^{+}+\mathrm{H}_{2} \mathrm{O} \rightarrow \mathrm{CH}_{3} \mathrm{OH}_{2}^{+}+h v$, followed by the electron recombination reaction $\mathrm{CH}_{3} \mathrm{OH}_{2}^{+}+\mathrm{e}^{-} \rightarrow \mathrm{CH}_{3} \mathrm{OH}+\mathrm{H}$. The latter reaction is, however, too inefficient to explain the observed $\mathrm{CH}_{3} \mathrm{OH}$ abundances in star-forming regions alone (see, e.g., Wirström et al. 2011, and references therein). For example, the $\mathrm{CH}_{3} \mathrm{OH}$ observations performed by van der Tak et al. (2000) towards a sample of 13 massive young stellar objects (MYSOs) suggested three types of abundance profiles: the coldest sources have $\mathrm{CH}_{3} \mathrm{OH}$ abundances of $\sim 10^{-9}$, warmer sources are characterised by a range of abundances from $\sim 10^{-9}$ to $\sim 10^{-7}$, and the hot cores have $x\left(\mathrm{CH}_{3} \mathrm{OH}\right) \sim 10^{-7}$. These high abundances indicate that solid $\mathrm{CH}_{3} \mathrm{OH}$ must have been evaporated from the icy grain mantles into the gas phase. In the grain-surface chemistry scheme, $\mathrm{CH}_{3} \mathrm{OH}$ is formed through successive hydrogenation of $\mathrm{CO}$ onto dust grains $\left(\mathrm{CO} \rightarrow \mathrm{HCO} \rightarrow \mathrm{H}_{2} \mathrm{CO} \rightarrow\right.$ $\mathrm{CH}_{2} \mathrm{OH} \rightarrow \mathrm{CH}_{3} \mathrm{OH}$ ), and subsequent grain mantle evaporation is then predicted to cause the gas-phase abundance of $\mathrm{CH}_{3} \mathrm{OH}$ to be $>10^{-8}$ (e.g., van der Tak et al. 2000; Charnley et al. 2004; Garrod et al. 2008). In addition to grain heating, methanol can be released from grain mantles by moderate shocks (e.g., Bachiller \& Perez Gutierrez 1997, and references therein).

The methanol abundances derived in the present work, that is the lowest values found in SMM 2, do not appear to necessarily require mantle evaporation (see below). The detection of high-velocity ${ }^{13} \mathrm{CO}$ wing emission in SMM 2, however, suggests the presence of outflow activity. The rest of the $x\left(\mathrm{CH}_{3} \mathrm{OH}\right)$ values, particularly in SMM 3 and IRAS 13037, appear to imply that $\mathrm{CH}_{3} \mathrm{OH}$ has been injected from dust grains into the gas. In SMM 3, the high $\mathrm{CH}_{3} \mathrm{OH}$ abundance together with the $\mathrm{SiO}$ detection and ${ }^{13} \mathrm{CO}$ wing emission, are likely related to outflow activity. The latter wing-emission signature was also found towards IRAS 13037, but the SED of this source is indicative of hot dust (Paper I). Therefore, in addition to grain processing in shocks, the central $\mathrm{YSO}(\mathrm{s})$ may have raised the dust temperature above $100 \mathrm{~K}$ in IRAS 13037, which is the approximate sublimation temperature of $\mathrm{CH}_{3} \mathrm{OH}$ (e.g., Garrod \& Herbst 2006). Interestingly, besides the highest $\mathrm{CH}_{3} \mathrm{OH}$ abundance, the strongest CO depletion was found towards SMM 3. This could be a sign of enhanced methanol production in SMM 3 caused by CO freeze-out (e.g., Whittet et al. 2011, and references therein); we still see a remnant of stronger $\mathrm{CO}$ depletion where $\mathrm{CO}$ hydrogenation on dust grains has occurred, as well as the product of this process, i.e., the gas-phase $\mathrm{CH}_{3} \mathrm{OH}$.

Additional $\mathrm{CH}_{3} \mathrm{OH}$ observations towards IRDCs have been done by other authors. Beuther \& Sridharan (2007) studied a sample of massive clumps within IRDCs, and measured $\mathrm{CH}_{3} \mathrm{OH}$ column densities and abundances of $1.7-37.2 \times 10^{13} \mathrm{~cm}^{-2}$ and $0.4-10.7 \times 10^{-10}$, respectively. Although their column densities are very similar to our values, our abundances are generally higher. As discussed by Beuther \& Sridharan (2007), their average $\mathrm{CH}_{3} \mathrm{OH}$ abundance of $4.3 \times 10^{-10}$ is similar to the $\mathrm{CH}_{3} \mathrm{OH}$ abundances of $6 \times 10^{-10}$ found in the low-mass starless cores L1498 and L1517B by Tafalla et al. (2006). There are no internal heating/outflows in starless cores, hence grain processing is unexpected in these sources. Sakai et al. (2010) derived $\mathrm{CH}_{3} \mathrm{OH}$ column densities in the range $<2.9-24.8 \times 10^{14} \mathrm{~cm}^{-2}$ towards a sample of 20 massive clumps within IRDCs, which are much higher than those found in the present work. Sakai et al. (2010) pointed out that their much higher $N\left(\mathrm{CH}_{3} \mathrm{OH}\right)$ values than to those of Beuther \& Sridharan (2007) could be due to the source selection criteria; SSH10 studied MSX-dark clumps with signatures of star-formation activity. On the basis of the observed correlation between the $\mathrm{CH}_{3} \mathrm{OH}$ and $\mathrm{SiO}$ abundances, SSH10 concluded that the origin of $\mathrm{CH}_{3} \mathrm{OH}$ in the MSX-dark clumps is related to shocks. Gómez et al. (2011) inferred $\mathrm{CH}_{3} \mathrm{OH}$ abundances of $\sim 4 \times 10^{-9}-3 \times 10^{-8}$ in the IRDC core G11.11$0.12 \mathrm{P} 1$, and also suggested that outflow desorption is responsible for the release of $\mathrm{CH}_{3} \mathrm{OH}$ from ice mantles. With comparable $\mathrm{CH}_{3} \mathrm{OH}$ abundances, this could also be the case in the MIR-dark clump SMM 3.

\subsubsection{DCN}

We detected the $J=3-2$ transition of DCN towards SMM 3. To our knowledge, this is the first reported detection of DCN in an IRDC (Sakai et al. 2012, detected the DNC isotopomer towards different IRDC clumps). The first detection of DCN in the interstellar medium was made by Jefferts et al. (1973) towards the Orion Nebula. Mangum et al. (1991) mapped the Orion-KL region in DCN, and they suggested that most of the gas-phase DCN could have been released from icy grain mantles. Both the DCN and DNC molecules are the major products of the association reaction $\mathrm{D}+\mathrm{CN}$ on grain surfaces (Hiraoka et al. 2006), and DCN can be thermally desorbed at $\sim 100 \mathrm{~K}$ (e.g., Albertsson et al. 2011). In SMM 3, we may see shock-originated DCN emission, which conforms to the results discussed above.

\section{Summary and conclusions}

We have carried out a molecular-line study of the IRDC G304.74 using the APEX telescope. All the clumps along the filamentary cloud were observed in $\mathrm{C}^{17} \mathrm{O}(2-1)$, and selected positions in the southern part of the filament were observed in the rotational lines of $\mathrm{SiO},{ }^{13} \mathrm{CO}$, and $\mathrm{CH}_{3} \mathrm{OH}$. The main results are summarised as follows:

1. The approximately 9 pc long filamentary IRDC G304.74 is a spatially coherent structure as indicated by the uniform $\mathrm{C}^{17} \mathrm{O}(2-1)$ radial velocities throughout the cloud.

2. The fragmentation of the filament into clumps appears to be caused by a "sausage"-type fluid instability, in agreement with the results for other IRDCs, such as the "Nessie" Nebula (Jackson et al. 2010). On smaller scales, however, clumps may have fragmented into smaller cores owing to, e.g., a Jeans-type instability. Studying this possibility would require higher-resolution observations.

3. Most of the clumps appear to be gravitationally bound $\left(\alpha_{\text {vir }} \lesssim 2\right.$ ), although we found evidence that the external pressure may play an important role in their overall dynamics. The ambient pressure could be due to the turbulent ram pressure, in agreement with the hypothesis that the formation of the whole filament is caused by converging supersonic turbulent flows (Paper I). 
4. The whole filament might be close to virial balance, as suggested by the analysis of the virial mass per unit length. Radial collapse could be retarded by the poloidal magnetic field component. Our clump-by-clump virial analysis implies that some of the clumps in the southern part of the filament may be warmer than the remaining clumps. This is consistent with our analysis of dust opacity ratios at $8 \mu \mathrm{m}$ and $870 \mu \mathrm{m}$ in Paper I, which suggested dust temperatures of $\sim 30 \mathrm{~K}$ in SMM 1 and 2, although with considerable uncertainties.

5. The clumps show trans- to supersonic non-thermal internal motions. Moreover, the observed clumps in the southern part of the filament show large-scale infall motion signatures in the ${ }^{13} \mathrm{CO}(2-1)$ line. The infall velocities and mass infall rates were estimated to be $\sim 0.03-0.20 \mathrm{~km} \mathrm{~s}^{-1}$ and $\sim 2-36 \times 10^{-5} M_{\odot} \mathrm{yr}^{-1}$, respectively.

6. None of the clumps clearly fulfill the mass-radius limit of massive-star formation proposed by Kauffmann \& Pillai (2010). The clumps may "only" form stellar clusters and/or intermediate-mass stars. Two IRAS sources in the cloud have luminosities $\sim 1.5-2 \times 10^{3} L_{\odot}$ (Paper I), which is consistent with the latter scenario.

7. The CO molecules do not appear to be significantly depleted in the clumps $\left(f_{\mathrm{D}} \lesssim 2\right)$. The star-formation activity, such as outflows, may have released $\mathrm{CO}$ from the icy grain mantles into the gas phase. Some of the ${ }^{13} \mathrm{CO}(2-1)$ lines also show broad wings of emission, suggesting the presence of outflowing gas.

8. We estimate that the fractional $\mathrm{CH}_{3} \mathrm{OH}$ abundances in the clumps are around $2 \times 10^{-10}-1 \times 10^{-8}$. No evidence of hotcore type methanol abundance of $\sim 10^{-7}$ was found among the observed five clumps. Outflow activity appears to be a likely explanation for the observed $\mathrm{CH}_{3} \mathrm{OH}$ abundance in the MIR-dark clump SMM 3. The presence of outflows in SMM 3 is also indicated by the detection of $\mathrm{SiO}$ and DCN emission towards this source.

Acknowledgements. I express my gratitude to the anonymous referee for his/her constructive comments and suggestions on the original manuscript. I wish to thank the staff at the APEX telescope for performing the service-mode observations presented in this paper. I would also like to thank the people who maintain the CDMS and JPL molecular spectroscopy databases, and the Splatalogue Database for Astronomical Spectroscopy. The Academy of Finland is acknowledged for the financial support through grant 132291. This research has made use of NASA's Astrophysics Data System and the NASA/IPAC Infrared Science Archive, which is operated by the JPL, California Institute of Technology, under contract with the NASA.

\section{Appendix A: Derivation of the physical and chemical properties of the cloud/clumps}

This appendix details the analysis and formulae discussed in Sects. 4.1-4.4.

\section{A.1. Kinematic distance}

To calculate the cloud kinematic distance, we adopted the mean $\mathrm{C}^{17} \mathrm{O}(2-1)$ radial velocity of $-26.7 \mathrm{~km} \mathrm{~s}^{-1}$. We also employed the rotation curve of Reid et al. (2009), which is based on measurements of trigonometric parallaxes and the proper motions of masers in high-mass star-forming regions. The best-fit rotation parameters of Reid et al. (2009) are $\left(\Theta_{0}, R_{0}\right)=\left(254 \mathrm{~km} \mathrm{~s}^{-1}\right.$, $8.4 \mathrm{kpc})$, where $R_{0}$ is the solar Galactocentric distance and $\Theta_{0}$ is the rotation velocity at $R_{0}$. The resulting near kinematic distance, as expected to be appropriate for an IRDC seen in absorption, is $d=2.54 \pm 0.66 \mathrm{kpc}$. The corresponding Galactocentric distance is about $R_{\mathrm{GC}} \simeq 7.26 \mathrm{kpc}$. Besides being an IRDC, the Galactic latitude of $\mathrm{G} 304.74, b=+1.32$, indeed supports the idea that it lies at the near distance, because then the cloud is $\sim 59 \mathrm{pc}$ above the Galactic plane. In the case of the far kinematic distance, which is $\sim 7.05 \mathrm{kpc}$, it would be suspiciously high above the Galactic plane, i.e., $\sim 162$ pc. This would be more than twice the scale height of the molecular disk, $z_{1 / 2} \sim 70 \mathrm{pc}$ (Bronfman et al. 1988; see also Fontani et al. 2005).

\section{A.2. Revision of clump properties presented in Paper I}

The revised cloud distance was used to recalculate the distancedependent clump parameters presented in Paper I, i.e., the clump radius $(R \propto d)$, mass $\left(M \propto d^{2}\right)$, and volume-averaged $\mathrm{H}_{2}$ number density $\left(\left\langle n\left(\mathrm{H}_{2}\right)\right\rangle \propto M / R^{3} \propto d^{-1}\right)$ (see Eqs. (6)-(8) in Paper I). We previously assumed a uniform dust temperature of $T_{\text {dust }}=15 \mathrm{~K}$ for all the other sources except IRAS 13037 and IRAS 13039 , for which we used the value $\simeq 22 \mathrm{~K}$ derived from their spectral energy distributions (SEDs; see Fig. 5 of Paper I). Here, we adopt these same temperature values but the assumed $15 \mathrm{~K}$ temperature is now assumed to have a $\pm 5 \mathrm{~K}$ uncertainty. This is expected to be a reasonable choice, because previous molecular-line observations of clumps within IRDCs have shown the typical gas kinetic temperature to lie in the range $T_{\text {kin }} \approx 10-20 \mathrm{~K}$ (Carey et al. 1998; Teyssier et al. 2002; Sridharan et al. 2005; Pillai et al. 2006; Sakai et al. 2008; Zhang et al. 2011; Devine et al. 2011; Ragan et al. 2011). Moreover, at high densities of $n\left(\mathrm{H}_{2}\right) \gtrsim 3 \times 10^{4} \mathrm{~cm}^{-3}$, where collisional coupling between the gas and dust becomes efficient, the gas and dust temperatures are expected to be similar (e.g., Galli et al. 2002). For comparison, Nguyên Luong et al. (2011) derived a mean dust temperature of $\sim 14.5 \mathrm{~K}$ for the clumps/cores in the filamentary IRDC G035.39-00.33. To estimate the errors in $R, M$, and $\left\langle n\left(\mathrm{H}_{2}\right)\right\rangle$, we took into account the temperature uncertainty, and the distance error of $\pm 0.66 \mathrm{kpc}$. The \pm 5 -K temperature error was also used to estimate the uncertainty in the $\mathrm{H}_{2}$ column density.

\section{A.3. Molecular column densities and fractional abundances}

The spectral line intensity can be expressed as the main-beam brightness temperature, which is given by

$T_{\mathrm{MB}}=\frac{h v}{k_{\mathrm{B}}}\left[F\left(T_{\mathrm{ex}}\right)-F\left(T_{\mathrm{bg}}\right)\right]\left(1-\mathrm{e}^{-\tau_{v}}\right)$,

where $h$ is the Planck constant, $k_{\mathrm{B}}$ is the Boltzmann constant, $T_{\mathrm{ex}}$ is the line excitation temperature, $T_{\mathrm{bg}}$ is the background temperature (taken to be the cosmic background radiation temperature of $2.725 \mathrm{~K}), \tau_{v}$ is the line optical thickness, and the function $F(T)$ is identical to the (average) photon occupation number

$F(T) \equiv \frac{1}{\mathrm{e}^{h v / k_{\mathrm{B}} T}-1}$.

We note that the often used Rayleigh-Jeans equivalent temperature is given by $J(T)=h v / k_{\mathrm{B}} \times F(T)$. From Eq. (A.1), we can solve for the peak optical thickness

$\tau_{0}=-\ln \left\{1-\frac{T_{\mathrm{MB}}}{\frac{h v}{k_{\mathrm{B}}}\left[F\left(T_{\mathrm{ex}}\right)-F\left(T_{\mathrm{bg}}\right)\right]}\right\}$.

Equation (A.3) was used to estimate the value of $\tau_{0}$ for the lines used in the column-density calculations (see Col. (7) of Table 3). 
Table A.1. Spectroscopic properties of the observed transitions.

\begin{tabular}{lcccccc}
\hline \hline Transition & $\begin{array}{c}n_{\text {crit }} \\
{\left[\mathrm{cm}^{-3}\right]}\end{array}$ & $\begin{array}{c}E_{\mathrm{u}} / k_{\mathrm{B}} \\
{[\mathrm{K}]}\end{array}$ & $\begin{array}{c}\mu_{\mathrm{el}}^{2} S \\
{\left[\mathrm{D}^{2}\right]}\end{array}$ & $\begin{array}{c}A \\
{[\mathrm{MHz}]}\end{array}$ & $\begin{array}{c}B \\
{[\mathrm{MHz}]}\end{array}$ & $\begin{array}{c}C \\
{[\mathrm{MHz}]}\end{array}$ \\
\hline $\mathrm{SiO}(5-4)$ & $2.3 \times 10^{6}$ & 31.3 & 48.14651 & $\ldots$ & 21711.96 & $\ldots$ \\
$\mathrm{DCN}(3-2)$ & $\sim 10^{7}$ & 20.9 & 80.50709 & $\ldots$ & 36207.46 & $\ldots$ \\
${ }^{13} \mathrm{CO}(2-1)$ & $8.9 \times 10^{3}$ & 15.9 & 0.02437 & $\ldots$ & 55101.01 & $\ldots$ \\
$\mathrm{C}^{17} \mathrm{O}(2-1)$ & $9.5 \times 10^{3}$ & 16.2 & 0.02432 & $\ldots$ & 56179.99 & $\ldots$ \\
$\mathrm{CH}_{3} \mathrm{OH}\left(5_{-1,5}-4_{-1,4}\right)-\mathrm{E}$ & $9.5 \times 10^{5}$ & 40.4 & 3.88240 & 127484.0 & 24679.98 & 23769.70 \\
$\mathrm{CH}_{3} \mathrm{OH}\left(5_{0,5}-4_{0,4}\right)-\mathrm{A}^{+}$ & $1.1 \times 10^{6}$ & 34.8 & 4.04297 & $-\|-$ & $-\|-$ & $-\|-$ \\
$\mathrm{CH}_{3} \mathrm{OH}\left(5_{4, *}-4_{4, *}\right)-\mathrm{A}^{+/-a}$ & $3.5 \times 10^{5}$ & 115.2 & 1.45278 & $-\|-$ & $-\|-$ & $-\|-$ \\
\hline
\end{tabular}

Notes. Column (2) gives the critical density of the transition, $n_{\text {crit }}=A_{\mathrm{ul}} / C_{\mathrm{ul}}$, where $A_{\mathrm{ul}}$ is the spontaneous decay rate and $C_{\mathrm{ul}}$ is the collisional de-excitation rate. It was calculated at $T=15 \mathrm{~K}$ using the collisional-rate data available in the Leiden Atomic and Molecular Database (LAMDA; http://www.strw. leidenuniv.nl/ moldata/) (Schöier et al. 2005). For DCN, there is no molecular datafile available, so we used the collisional rate coefficient of $\mathrm{HCN}$ to estimate $n_{\text {crit }}$. Columns (3) and (4) give the upper-state energy and the product $\mu_{\mathrm{el}}^{2} S$, where $\mu_{\mathrm{el}}$ is the permanent electric dipole moment, and $S$ is the line strength. Columns (5)-(7) list the rotational constants of the molecules. The data were compiled from

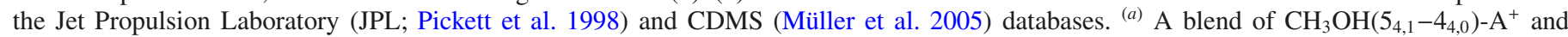
$\mathrm{CH}_{3} \mathrm{OH}\left(5_{4,2}-4_{4,1}\right)-\mathrm{A}^{-}$, which have the same spectroscopic parameters.

The assumptions made concerning the value of $T_{\mathrm{ex}}$ are described below.

The beam-averaged column densities, $N(\mathrm{~mol})$, of $\mathrm{C}^{17} \mathrm{O}, \mathrm{SiO}$, $\mathrm{CH}_{3} \mathrm{OH}$, and DCN were calculated under the assumption of local thermodynamic equilibrium (LTE), and assuming optically thin emission. The latter assumption seems to be valid for our lines. In this case, $N(\mathrm{~mol})$ is directly proportional to the integrated line intensity as

$N(\mathrm{~mol})=\frac{3 k_{\mathrm{B}} \epsilon_{0}}{2 \pi^{2}} \frac{1}{v \mu_{\mathrm{el}}^{2} S} \frac{Z_{\mathrm{rot}}\left(T_{\mathrm{ex}}\right)}{g_{K} g_{I}} \frac{\mathrm{e}^{E_{\mathrm{u}} / k_{\mathrm{B}} T_{\mathrm{ex}}}}{1-\frac{F\left(T_{\mathrm{bg}}\right)}{F\left(T_{\mathrm{ex}}\right)}} \int T_{\mathrm{MB}} \mathrm{d} v$,

where $\epsilon_{0}$ is the vacuum permittivity, $\mu_{\mathrm{el}}$ is the permanent electric dipole moment, $S$ is the line strength, $Z_{\text {rot }}$ is the rotational partition function, $g_{K}$ is the $K$-level degeneracy, $g_{I}$ is the reduced nuclear spin degeneracy (see, e.g., Turner 1991), and $E_{\mathrm{u}}$ is the energy of the upper-transition state. We note that in the derivation of Eq. (A.4), the electric dipole moment matrix element is defined to be $\left|\mu_{\mathrm{ul}}\right|^{2}=\mu_{\mathrm{el}}^{2} S / g_{\mathrm{u}}$, where $g_{\mathrm{u}}=2 J+1$ is the rotational degeneracy of the upper state (Townes \& Schawlow 1975). For linear molecules, we note that $g_{K}=g_{I}=1$ for all levels, whereas for $A$-type $\mathrm{CH}_{3} \mathrm{OH}, g_{K}=1$ and $g_{I}=2$, and for $E$-type $\mathrm{CH}_{3} \mathrm{OH}, g_{K}=2$ and $g_{I}=1$ (Turner 1991). We derived the $\mathrm{CH}_{3} \mathrm{OH}$ column densities from the integrated intensity of both the $\mathrm{CH}_{3} \mathrm{OH}\left(5_{0,5}-4_{0,4}\right)-\mathrm{A}^{+}$and $\mathrm{CH}_{3} \mathrm{OH}\left(5_{-1,5}-4_{-1,4}\right)$-E lines, and the results were found to be comparable to each other. In this paper, we only give the $N\left(\mathrm{CH}_{3} \mathrm{OH}\right)$ values derived from the above $\mathrm{A}^{+}$-transition. We note that we detected too few lines to employ the population diagram analysis to derive the rotational temperature and column density of $\mathrm{CH}_{3} \mathrm{OH}$ (Goldsmith \& Langer 1999).

For the linear molecules $\mathrm{C}^{17} \mathrm{O}, \mathrm{SiO}$, and $\mathrm{DCN}$, we approximated the partition function as

$Z_{\mathrm{rot}}\left(T_{\mathrm{ex}}\right) \simeq \frac{k_{\mathrm{B}} T_{\mathrm{ex}}}{h B}+\frac{1}{3}$

where $B$ is the rotational constant. The above expression is appropriate for heteropolar molecules at the high temperature limit $\left(h B / k_{\mathrm{B}} T_{\mathrm{ex}} \ll 1\right)$. The partition function of methanol, which is a slightly asymmetric rotor with one internal rotor and $A$ - and $E$-symmetry states, was calculated as

$Z_{\mathrm{rot}}\left(T_{\mathrm{ex}}\right)=Z_{\mathrm{rot}}(A)+Z_{\mathrm{rot}}(E)=2 \sqrt{\frac{\pi\left(k_{\mathrm{B}} T_{\mathrm{ex}}\right)^{3}}{h^{3} A B C}}$, where $A, B$, and $C$ are the rotational constants (Turner 1991). The spectroscopic properties of the observed molecules/transitions are given in Table A.1

The unknown parameter in the above optical-thickness and column-density calculations is the excitation temperature, $T_{\mathrm{ex}}$. In the case of $\mathrm{C}^{17} \mathrm{O}$, we assumed that the line is thermalised at the clump temperature, and adopted the value $T_{\mathrm{ex}}=15 \pm 5 \mathrm{~K}$ for all the clumps except the IRAS sources 13037 and 13039, for which the value $T_{\mathrm{ex}}=22 \mathrm{~K}$ was used (Appendix A.2). This is likely to be true for our clumps, because even their average $\mathrm{H}_{2}$ densities are comparable to the critical density of $\mathrm{C}^{17} \mathrm{O}(2-1)\left(9.5 \times 10^{3} \mathrm{~cm}^{-3}\right.$; see Col. (2) of Table A.1). Fontani et al. (2005) derived the $\mathrm{C}^{17} \mathrm{O}$ rotational excitation temperature towards IRAS 13039 of $18 \mathrm{~K}$, which is very close to the value we adopted. The approximate-equality $T_{\text {kin }} \simeq T_{\text {ex }}\left[\mathrm{C}^{17} \mathrm{O}(2-1)\right]$ was derived by Miettinen et al. (2011) for a sample of clumps associated with IRDCs. For the linear rotors $\mathrm{SiO}$ and DCN, we assumed that $T_{\mathrm{ex}}$ is in the range $\left[5 \mathrm{~K}, E_{\mathrm{u}} / k_{\mathrm{B}}\right]$, whereas for $\mathrm{CH}_{3} \mathrm{OH}$ it was assumed that $T_{\mathrm{ex}} \in\left[10 \mathrm{~K}, 2 E_{\mathrm{u}} / 3 k_{\mathrm{B}}\right]$. The adopted upper $T_{\text {ex }}$ limits result from the turning point of the temperaturedependent part of $N(\mathrm{~mol})$, i.e., from the derivative

$\frac{\mathrm{d} N(\mathrm{~mol})}{\mathrm{d} T_{\mathrm{ex}}}=\frac{\mathrm{d}}{\mathrm{d} T_{\mathrm{ex}}} Z_{\mathrm{rot}}\left(T_{\mathrm{ex}}\right) \mathrm{e}^{E_{\mathrm{u}} / k_{\mathrm{B}} T_{\mathrm{ex}}}=0$,

where it is assumed that $T_{\mathrm{ex}} \gg T_{\mathrm{bg}}$ (e.g., Hatchell et al. 1998). The upper $T_{\mathrm{ex}}$ limits thus derived lead to the lower limit to $N(\mathrm{~mol})$. We note that the $T_{\mathrm{ex}}\left(\mathrm{CH}_{3} \mathrm{OH}\right)$ range we used, 10-23.2 K, is comparable to the $\mathrm{CH}_{3} \mathrm{OH}\left(2_{k}-1_{k}\right)$ rotational temperatures of $\sim 13.4-24.5 \mathrm{~K}$ derived by SSH10 towards a sample of massive clumps within IRDCs.

We calculated the fractional abundances of the molecules by dividing the molecular column density by the $\mathrm{H}_{2}$ column density: $x(\mathrm{~mol})=N(\mathrm{~mol}) / N\left(\mathrm{H}_{2}\right)$. For this purpose, the values of $N\left(\mathrm{H}_{2}\right)$ were derived from the LABOCA dust continuum map smoothed to the corresponding resolution of the line observations.

\section{A.4. CO depletion factors}

Since the clumps under investigation (or at least most of them) are presumably cold, the $\mathrm{CO}$ molecules should efficiently freeze 
out onto dust grain surfaces. To estimate the extent of this $\mathrm{CO}$ depletion, we calculated the $\mathrm{CO}$ depletion factors, $f_{\mathrm{D}}$, following the analysis presented in the paper by Fontani et al. (2006). If $x(\mathrm{CO})_{\text {can }}$ is the "canonical" (undepleted) abundance of $\mathrm{CO}$, and $x(\mathrm{CO})_{\text {obs }}$ is the observed $\mathrm{CO}$ abundance, $f_{\mathrm{D}}$ is given by

$f_{\mathrm{D}}=\frac{x(\mathrm{CO})_{\mathrm{can}}}{x(\mathrm{CO})_{\mathrm{obs}}}$

The "canonical" CO abundance at the Galactocentric distance $R_{\mathrm{GC}}$ was calculated using the relationship (Eq. (7) in Fontani et al. 2006)

$$
x(\mathrm{CO})_{\text {can }}=9.5 \times 10^{-5} \mathrm{e}^{1.105-0.13 R_{\mathrm{GC}}[\mathrm{kpc}]} .
$$

We note that this relationship assumes that $R_{0}=8.5 \mathrm{kpc}$, whereas our $R_{\mathrm{GC}}$ value was computed using $R_{0}=8.4 \mathrm{kpc}$. This small discrepancy is however negligible. At $R_{\mathrm{GC}}=8.5 \mathrm{kpc}$, the above relationship gives the standard value $9.5 \times 10^{-5}$ for the abundance of the main CO isotopologue in the solar neighbourhood (Frerking et al. 1982). At the Galactocentric distance of G304.74, $x(\mathrm{CO})_{\text {can }} \simeq 1.1 \times 10^{-4}$. To calculate the "canonical" $\mathrm{C}^{17} \mathrm{O}$ abundance we employ the oxygen-isotopic ratio (Wilson $\&$ Rood 1994)

$$
\frac{\left[{ }^{16} \mathrm{O}\right]}{\left[{ }^{18} \mathrm{O}\right]}=58.8 \times R_{\mathrm{GC}}[\mathrm{kpc}]+37.1 \text {, }
$$

and the ratio $\left[{ }^{18} \mathrm{O}\right] /\left[{ }^{17} \mathrm{O}\right]=3.52$ (Frerking et al. 1982)

$$
\begin{aligned}
x\left(\mathrm{C}^{17} \mathrm{O}\right)_{\mathrm{can}} & =\frac{x(\mathrm{CO})_{\mathrm{can}}}{\left[{ }^{18} \mathrm{O}\right] /\left[{ }^{17} \mathrm{O}\right] \times\left[{ }^{16} \mathrm{O}\right] /\left[{ }^{18} \mathrm{O}\right]} \\
& =\frac{x(\mathrm{CO})_{\mathrm{can}}}{3.52 \times\left(58.8 \times R_{\mathrm{GC}}[\mathrm{kpc}]+37.1\right)} .
\end{aligned}
$$

For $\mathrm{G} 304.74$, we get $x\left(\mathrm{C}^{17} \mathrm{O}\right)_{\mathrm{can}} \simeq x(\mathrm{CO})_{\mathrm{can}} / 1633 \simeq 6.8 \times$ $10^{-8}$. The depletion factor $f_{\mathrm{D}}$ is then calculated from $f_{\mathrm{D}}=$ $x\left(\mathrm{C}^{17} \mathrm{O}\right)_{\mathrm{can}} / x\left(\mathrm{C}^{17} \mathrm{O}\right)_{\mathrm{obs}}$.

\section{References}

Albertsson, T., Semenov, D. A., \& Henning, T. 2011, ApJ, submitted [arXiv: 1110.2644]

André, P., Men'shchikov, A., Bontemps, S., et al. 2010, A\&A, 518, L102

Bachiller, R., \& Perez Gutierrez, M. 1997, ApJ, 487, L93

Ballesteros-Paredes, J. 2006, MNRAS, 372, 443

Ballesteros-Paredes, J., Vázquez-Semadeni, E., \& Scalo, J. 1999, ApJ, 515, 286

Ballesteros-Paredes, J., Hartmann, L. W., Vázquez-Semadeni, E., et al. 2011, MNRAS, 411, 65

Bastien, P. 1983, A\&A, 119, 109

Belitsky, V., Lapkin, I., Vassilev, V., et al. 2007, in Proc. joint 32nd International Conference on Infrared Millimeter Waves and 15th International Conference on Terahertz Electronics, September 3-7, 2007 (Cardiff, Wales, UK: City Hall), 326

Beltrán, M. T., Brand, J., Cesaroni, R., et al. 2006, A\&A, 447, 221

Beltrán, M. T., Cesaroni, R., Neri, R., \& Codella, C. 2011, A\&A, 525, A151

Bertoldi, F., \& McKee, C. F. 1992, ApJ, 395, 140 (BM92)

Beuther, H., \& Sridharan, T. K. 2007, ApJ, 668, 348

Beuther, H., Schilke, P., Menten, K. M., et al. 2002, ApJ, 566, 945

Beuther, H., Kainulainen, J., Henning, T., et al. 2011, A\&A, 533, A17

Bonazzola, S., Heyvaerts, J., Falgarone, E., et al. 1987, A\&A, 172, 293

Bonnell, I. A., \& Bate, M. R. 2006, MNRAS, 370, 488

Brand, J., \& Blitz, L. 1993, A\&A, 275, 67

Bronfman, L., Cohen, R. S., Alvarez, H., et al. 1988, ApJ, 324, 248

Carey, S. J., Clark, F. O., Egan, M. P., et al. 1998, ApJ, 508, 721

Carey, S. J., Feldman, P. A., Redman, R. O., et al. 2000, ApJ, 543, L157

Cazzoli, G., Puzzarini, C., \& Lapinov, A. V. 2004, ApJ, 611, 615

Chandrasekhar, S. 1951, Proc. R. Soc. Lond. A, 210, 26

Chandrasekhar, S., \& Fermi, E. 1953, ApJ, 118, 116

Charnley, S. B., Ehrenfreund, P., Millar, T. J., et al. 2004, MNRAS, 347, 157

Chen, X., Shen, Z.-Q., Li, J.-J., et al. 2010, ApJ, 710, 150
Chen, H.-R., Liu, S.-Y., Su, Y.-N., \& Wang, M.-Y. 2011, ApJ, 743, 196 Curry, C. L. 2000, ApJ, 541, 831

Davies, B., Hoare, M. G., Lumsden, S. L., et al. 2011, MNRAS, 416, 972

Devine, K. E., Chandler, C. J., Brogan, C., et al. 2011, ApJ, 733, 44

Egan, M. P., Shipman, R. F., Price, S. D., et al. 1998, ApJ, 494, L199

Evans, N. J., II 1999, ARA\&A, 37, 311

Fiege, J. D., \& Pudritz, R. E. 2000a, MNRAS, 311, 85

Fiege, J. D., \& Pudritz, R. E. 2000b, MNRAS, 311, 105

Fiege, J. D., Johnstone, D., Redman, R. O., \& Feldman, P. A. 2004, ApJ, 616, 925

Field, G. B., Blackman, E. G., \& Keto, E. R. 2011, MNRAS, 416, 710

Fontani, F., Beltrán, M. T., Brand, J., et al. 2005, A\&A, 432, 921

Fontani, F., Caselli, P., Crapsi, A., et al. 2006, A\&A, 460, 709

Frerking, M. A., Langer, W. D., \& Wilson, R. W. 1982, ApJ, 262, 590

Fuller, G. A., \& Myers, P. C. 1992, ApJ, 384, 523

Galli, D., Walmsley, M., \& Gonçalves, J. 2002, A\&A, 394, 275

Garrod, R. T., \& Herbst, E. 2006, A\&A, 457, 927

Garrod, R. T., Weaver, S. L. W., \& Herbst, E. 2008, ApJ, 682, 283

Goldsmith, P. F., \& Langer, W. D. 1999, ApJ, 517, 209

Gómez, L., Wyrowski, F., Pillai, T., et al. 2011, A\&A, 529, A161

Güsten, R., Nyman, L. A., Schilke, P., et al. 2006, A\&A, 454, L13

Hartquist, T. W., Dalgarno, A., \& Oppenheimer, M. 1980, ApJ, 236, 182

Hatchell, J., Thompson, M. A., Millar, T. J., \& MacDonald, G. H. 1998, A\&AS, 133,29

Heitsch, F., Hartmann, L. W., Slyz, A. D., et al. 2008, ApJ, 674, 316

Heitsch, F., Ballesteros-Paredes, J., \& Hartmann, L. 2009, ApJ, 704, 1735

Heitsch, F., Naab, T., \& Walch, S. 2011, MNRAS, 415, 271

Hennemann, M., Birkmann, S. M., Krause, O., et al. 2009, ApJ, 693, 1379

Henning, T., Linz, H., Krause, O., et al. 2010, A\&A, 518, L95

Hernandez, A. K., Tan, J. C., Caselli, P., et al. 2011, ApJ, 738, 11

Hiraoka, K., Ushiama, S., Enoura, T., et al. 2006, ApJ, 643, 917

Inutsuka, S.-I., \& Miyama, S. M. 1992, ApJ, 388, 392

Jackson, J. M., Finn, S. C., Chambers, E. T., et al. 2010, ApJ, 719, L185

Jefferts, K. B., Penzias, A. A., \& Wilson, R. W. 1973, ApJ, 179, L57

Jiménez-Serra, I., Caselli, P., Tan, J. C., et al. 2010, MNRAS, 406, 187

Johnstone, D., Fiege, J. D., Redman, R. O., et al. 2003, ApJ, 588, L37

Kainulainen, J., Beuther, H., Banerjee, R., et al. 2011a, A\&A, 530, A64

Kainulainen, J., Alves, J., Beuther, H., et al. 2011b, A\&A, 536, A48

Kang, M., Choi, M., Bieging, J. H., et al. 2011, ApJ, 743, 198

Kauffmann, J., \& Pillai, T. 2010, ApJ, 723, L7

Klaassen, P. D., \& Wilson, C. D. 2007, ApJ, 663, 1092

Klein, B., Philipp, S. D., Krämer, I., et al. 2006, A\&A, 454, L29

Klessen, R. S. 2001, ApJ, 556, 837

Klessen, R. S., \& Burkert, A. 2000, ApJS, 128, 287

Lada, C. J., Muench, A. A., Rathborne, J., et al. 2008, ApJ, 672, 410

Ladd, E. F., Fuller, G. A., \& Deane, J. R. 1998, ApJ, 495, 871

Leurini, S., Pillai, T., Stanke, T., et al. 2011, A\&A, 533, A85

Lucas, R. 1976, A\&A, 46, 473

Mangum, J. G., Plambeck, R. L., \& Wootten, A. 1991, ApJ, 369, 169

McKee, C. F., \& Zweibel, E. G. 1992, ApJ, 399, 551

McKee, C. F., \& Tan, J. C. 2003, ApJ, 585, 850

Miettinen, O., \& Harju, J. 2010, A\&A, 520, A102 (Paper I)

Miettinen, O., Hennemann, M., \& Linz, H. 2011, A\&A, 534, A134

Müller, H. S. P., Schlöder, F., Stutzki, J., \& Winnewisser, G. 2005, J. Mol. Struct., 742,215

Myers, P. C. 2009, ApJ, 700, 1609

Myers, P. C., Ladd, E. F., \& Fuller, G. A. 1991, ApJ, 372, L95

Myers, P. C., Mardones, D., Tafalla, M., et al. 1996, ApJ, 465, L133

Nagasawa, M. 1987, Prog. Theor. Phys., 77, 635

Nakajima, Y., \& Hanawa, T. 1996, ApJ, 467, 321

Nakamura, F., Hanawa, T., \& Nakano, T. 1993, PASJ, 45, 551

Nguyên Luong, Q., Motte, F., Hennemann, M., et al. 2011, A\&A, 535, A76

Ostriker, J. 1964, ApJ, 140, 1056

Pérault, M., Omont, A., Simon, G., et al. 1996, A\&A, 315, L165

Peretto, N., \& Fuller, G. A. 2009, A\&A, 505, 405

Pickett, H. M., Poynter, I. R. L., Cohen, E. A., et al. 1998, J. Quant. Spec. Radiat. Transf., 60, 883

Pillai, T., Wyrowski, F., Carey, S. J., et al. 2006, A\&A, 450, 569

Pound, M. W., \& Blitz, L. 1993, ApJ, 418, 328

Ragan, S. E., Bergin, E. A., \& Gutermuth, R. A. 2009, ApJ, 698, 324

Ragan, S. E., Bergin, E. A., \& Wilner, D. 2011, ApJ, 736, 163

Rathborne, J. M., Jackson, J. M., Chambers, E. T., et al. 2005, ApJ, 630, L181

Rathborne, J. M., Jackson, J. M., \& Simon, R. 2006, ApJ, 641, 389

Rathborne, J. M., Jackson, J. M., Zhang, Q., \& Simon, R. 2008, ApJ, 689, 1141

Reid, M. J., Menten, K. M., Zheng, X. W., et al. 2009, ApJ, 700, 137

Sakai, T., Sakai, N., Kamegai, K., et al. 2008, ApJ, 678, 1049

Sakai, T., Sakai, N., Hirota, T., \& Yamamoto, S. 2010, ApJ, 714, 1658 (SSH10)

Sakai, T., Sakai, N., Furuya, K., et al. 2012, ApJ, accepted [arXiv: 1201.4430] 
Schilke, P., Walmsley, C. M., Pineau des Forêts, G., \& Flower, D. R. 1997, A\&A, 321,293

Schöier, F. L., van der Tak, F. F. S., van Dishoeck, E. F., \& Black, J. H. 2005, A\&A, 432, 369

Simon, R., Jackson, J. M., Rathborne, J. M., \& Chambers, E. T. 2006, ApJ, 639, 227

Sridharan, T. K., Beuther, H., Saito, M., et al. 2005, ApJ, 634, L57

Stodólkiewicz, J. S. 1963, Acta Astron., 13, 30

Tafalla, M., Santiago-García, J., Myers, P. C., et al. 2006, A\&A, 455, 577

Teyssier, D., Hennebelle, P., \& Pérault, M. 2002, A\&A, 382, 624

Townes, C. H., \& Schawlow, L. S. 1975, Microwave Spectroscopy (New York:

Dover Publications, Inc.)

Turner, B. E. 1991, ApJS, 76, 617 van der Tak, F. F. S., van Dishoeck, E. F., \& Caselli, P. 2000, A\&A, 361, 327

Vassilev, V., Meledin, D., Lapkin, I., et al. 2008a, A\&A, 490, 1157

Vassilev, V., Henke, D., Lapkin, I., et al. 2008b, IEEE Microwave and Wireless Components Letters, 18, 1, 55

Wang, K., Zhang, Q., Wu, Y., \& Zhang, H. 2011, ApJ, 735, 64

Whittet, D. C. B., Cook, A. M., Herbst, E., et al. 2011, ApJ, 742, 28

Williams, J. P., de Geus, E. J., \& Blitz, L.1994, ApJ, 428, 693

Wilson, T. L., \& Rood, R. 1994, ARA\&A, 32, 191

Wirström, E. S., Geppert, W. D., Hjalmarson, Å., et al. 2011, A\&A, 533, A24

Zapata, L. A., Palau, A., Ho, P. T. P., et al. 2008, A\&A, 479, L25

Zhang, Q., Wang, Y., Pillai, T., \& Rathborne, J. 2009, ApJ, 696, 268

Zhang, S. B., Yang, J., Xu, Y., et al. 2011, ApJS, 193, 10

Zhou, S., Evans, N. J., II, Koempe, C., \& Walmsley, C. M. 1993, ApJ, 404, 232 